\title{
WestVirginiaUniversity
}

THE RESEARCH REPOSITORY @ WVU

Graduate Theses, Dissertations, and Problem Reports

2012

\section{Role of Chaperonin CCT in G-protein Biosynthesis}

\author{
Catherine Woodard \\ West Virginia University
}

Follow this and additional works at: https://researchrepository.wvu.edu/etd

\section{Recommended Citation}

Woodard, Catherine, "Role of Chaperonin CCT in G-protein Biosynthesis" (2012). Graduate Theses, Dissertations, and Problem Reports. 656.

https://researchrepository.wvu.edu/etd/656

This Thesis is protected by copyright and/or related rights. It has been brought to you by the The Research Repository @ WVU with permission from the rights-holder(s). You are free to use this Thesis in any way that is permitted by the copyright and related rights legislation that applies to your use. For other uses you must obtain permission from the rights-holder(s) directly, unless additional rights are indicated by a Creative Commons license in the record and/ or on the work itself. This Thesis has been accepted for inclusion in WVU Graduate Theses, Dissertations, and Problem Reports collection by an authorized administrator of The Research Repository @ WVU. For more information, please contact researchrepository@mail.wvu.edu. 


\title{
Role of Chaperonin CCT in G-protein Biosynthesis
}

\author{
Catherine Woodard
}

Thesis submitted to the College of Health Sciences at West Virginia University in partial fulfillment of the requirements for the degree of

Master of Science in

Biomedical Science

\author{
Maxim Sokolov, Ph.D., Chair \\ Visvanathan Ramamurthy, Ph.D. \\ Peter Stoilov, Ph.D. \\ Peter Mathers, Ph.D. \\ Eric Tucker, Ph.D. \\ Department of Biochemistry
}

Morgantown, West Virginia

2012 


\title{
ABSTRACT \\ Role of Chaperonin CCT in G-protein Biosynthesis
}

\author{
Catherine A. Woodard
}

Chaperonins are ubiquitous molecular chaperones that are found in all animal kingdoms. They all share a common structure and function to assist the folding of other proteins. All chaperonins consist of two stacked rings, which come together to form a central cavity where folding can take place (1). The eukaryotic cytosolic chaperonin containing TCP1 (CCT) is a member of the group II chaperonins, which are defined as having octameric or nonameric rings composed of more than one type of subunit (2). CCT is unique because it is composed of eight different subunits (CCT $\alpha$, $\beta, \gamma, \delta, \varepsilon, \zeta, \eta, \theta)(2)$, which also makes it the most complex of the group II chaperonins. The molecular diversity in the subunit composition of CCT gives rise to a more specialized role in protein folding than the general, nonspecific mechanism that is seen in other chaperonins. Substrate recognition is accomplished by the apical domains, which are highly flexible and undergo large conformational changes during the functional cycle. In the open conformation, the apical domains allow substrates to enter into central cavity. Nucleotide binding at the equatorial domain occurs once substrate is bound and ATP hydrolysis triggers the conversion from the open to the closed conformation (1). The exact mechanism has not been clearly defined, but is thought to involve a set of sequential, hierarchical steps.

CCT is an essential part of eukaryotic cell function, and it has been estimated that it folds up to $10 \%$ of newly synthesized cytosolic proteins (3). Some of the proteins that have been shown to depend on CCT for their biogenesis include cytoskeletal proteins ( $\alpha$-, and $\beta$-actin, $\alpha-, \beta-$, and $\gamma$-tubulin), histone deacetylases (HDAC3, Set3p, and Hos $2 p$ ), cell cycle regulators (Cdc20p, and Cdc55p), and proteins possessing the seven-bladed $\beta$-propeller WD40 domain, including the $\beta$-subunit of the heterotrimeric guanine nucleotide-binding protein (G-protein) complex $(4,5)$. Heterotrimeric $\mathrm{G}$ proteins are a highly conserved group of molecules involved in a great number of signaling processes. They are composed of three subunits $(\alpha-, \beta-$, and $\gamma)$, which are bound together in the inactive form. In this heterotrimeric state, guanosine di-phosphate (GDP) is bound to the G $\alpha$-subunit. Upon activation, the $\alpha$-subunit exchanges GDP for guanosine tri-phosphate (GTP). This exchange triggers $G \beta \gamma$ to dissociate from $\mathrm{G} \alpha$ and both $\mathrm{G} \alpha$-GTP and $\mathrm{G} \beta \gamma$ are then free to activate downstream effectors. This form of signal transduction is so common that G-proteins regulate or modulate nearly every cellular and physiological process. Because it is so critical for normal cellular function, dysregulation of G-protein signaling is associated with many human diseases (6) and more than half of the current pharmaceutical therapies target a component of the G-protein pathway (7). While much is known about the signal transduction, far less is known about the biogenesis and assembly of G-proteins.

The overall objective of my research has been to elucidate the mechanisms of the chaperonin containing Tailless Complex Polypeptide 1 (CCT) with respect to heterotrimeric guanine nucleotide-binding protein (G protein) signaling. The CCT co-factor, phosducin-like protein (PhLP) is required for G $\beta$ biosynthesis, and therefore its mechanism is critical for our understanding of this process. In the first chapter of my thesis, I take a structure-based approach to investigate the interaction between PhLP and G $\beta$. Currently there are no available structures of this complex, nor is there a single published structure of PhLP. Therefore, I used a homology modeling approach to generate the first known 3D structure of PhLP. Then in order to gain insight into this interaction, I docked PhLP to the G $\beta \gamma$ and compared it to the crystal structure of phosducin (Pdc), a closely related protein that also forms a complex with G $\beta \gamma$. The second chapter is centered on a truncated splice isoform of PhLP, referred to as PhLP Рновт (PhLPs), which has been shown to be a potent inhibitor of CCT. A recent paper published by my lab showed that PhLPs expression led to a decrease in $\alpha-, \beta-$, and $\gamma$-subunits of the G-protein transducin at the protein level. Later experiments showed that this decrease occurred at the mRNA level as well. While the decrease in $G \beta$ and $G \gamma$ wasn't surprising, the decrease in $G \alpha$ was unexpected since it is not a known substrate of CCT. In order to find a connection between CCT and $\mathrm{G} \alpha$, I looked for any perturbations of gene expression that occurred in transgenic mice constitutively expressing PhLPs using microarray results. Furthermore, because these mice express PhLPs at an early age I repeated these experiments in inducible transgenic mouse lines that we developed to express PhLPs in adult mice. Taken together, my research provides additional data to refine the mechanism of G $\beta$ biosynthesis and suggests that there is an additional level of regulatory control over G-proteins that connects the expression of all three subunits. 


\section{TABLE OF CONTENTS}

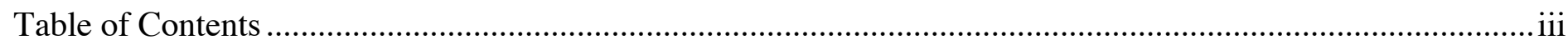

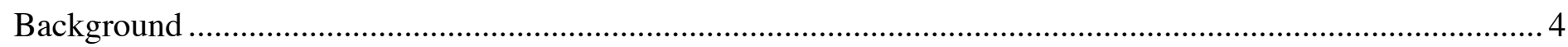

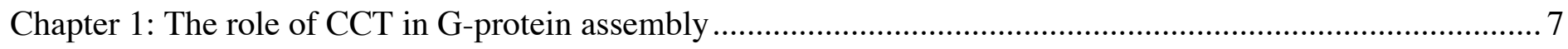

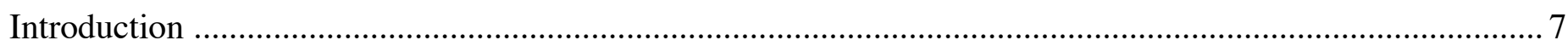

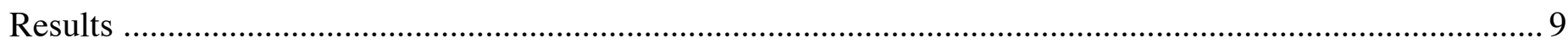

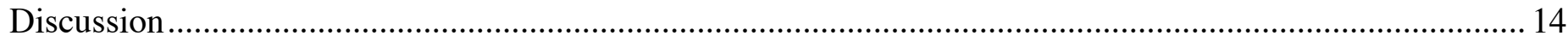

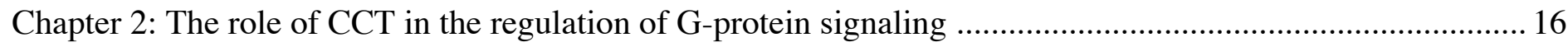

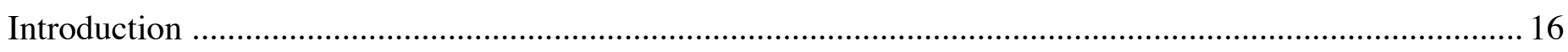

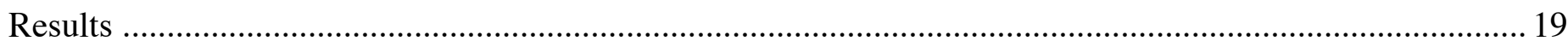

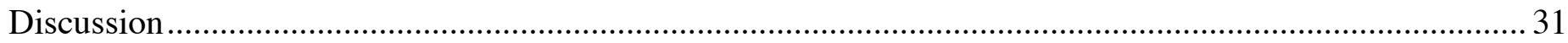

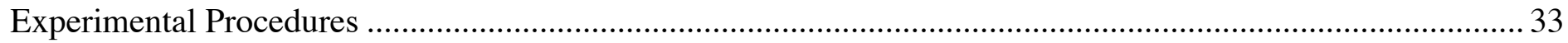

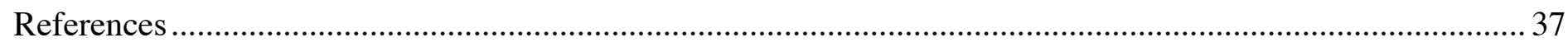




\section{BACKGROUND}

Although the eukaryotic cytosolic chaperonin containing TCP1 (CCT) is the most complex of the group II chaperonins, it is evolutionarily conserved and found in all eukaryotes (2). Figure 1 shows CCT's subunits each with a different color from a top view (Figure 1A), and a side view (Figure 1B \& C). The structure of CCT remained unresolved for many years due to instability of the complex. Early cryo-EM experiments demonstrated that the two hetero-octameric rings of CCT formed the typical cylindrical shape, but the resolution was not sufficient to observe secondary structures (8). An early model of CCT shows the different domains form the inner cavity, but incorrectly places the hetero-octameric rings stacked directly on top of one another (Figure 1D). More recent models have been refined using data from cryo-EM and X-ray crystallography studies at greater resolution to show an inter-ring misalignment that relates the upper and lower ring by a pseudo-two-fold symmetry (1)(Figure 1E). Figure 1E also illustrates two tubulin substrate molecules in the inner cavity (magenta and lime green). The molecular diversity in the subunit composition of CCT gives rise to a more specialized role in protein folding than the general, nonspecific mechanism that is seen in other chaperonins. Substrate recognition is accomplished by the apical domains (Figure 1D), which are highly flexible and undergo large conformational changes during the functional cycle. In the open conformation, the apical domains allow substrates to enter into central cavity. Nucleotide binding at the equatorial domain occurs once substrate is bound and ATP hydrolysis triggers the conversion from the open to the closed conformation (1). The exact mechanism has not been clearly defined, but is thought to involve a set of sequential, hierarchical steps.

It has been estimated that CCT may fold up to $10 \%$ of newly synthesized cytosolic proteins (3), so it plays an essential role in eukaryotic cellular function. Because CCT substrates are very diverse in function as well as structure, they cannot be predicted by homology alone (5). Some of the proteins that have been shown to depend on CCT for their biogenesis include cytoskeletal proteins ( $\alpha$-, and $\beta$-actin, $\alpha-, \beta$-, and $\gamma$-tubulin), histone deacetylases (HDAC3, Set3p, and Hos2p), cell cycle regulators (Cdc20p, and Cdc55p), and proteins possessing a seven-bladed $\beta$-propeller (WD40 repeat motif) $(4,5)$, such as the $\beta$-subunit of heterotrimeric guanine nucleotide-binding proteins (G-proteins).

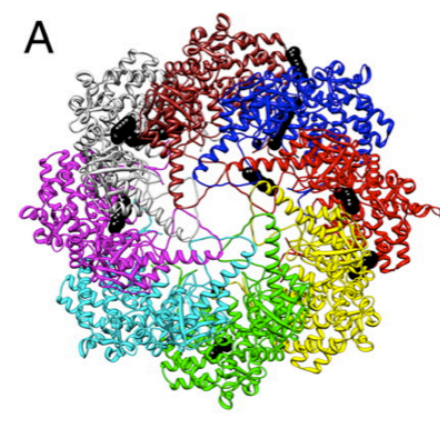

Figure 1: Structure of the Chaperonin Containing TComplex Polypeptide 1 (CCT). Each subunit of the octameric ring has been given a different color to illustrate the molecular diversity of CCT, A. top view, B. side view showing the pseudo-two fold symmetry of the two stacked rings, $\mathrm{C}$. the two octameric rings in $B$ have been separated and each subunit is labeled. D. An early model of CCT depicts the general domain structure, and $E$. a side view of a later model of CCT shows two molecules of tubulin (lime green and magenta) in the inner chamber

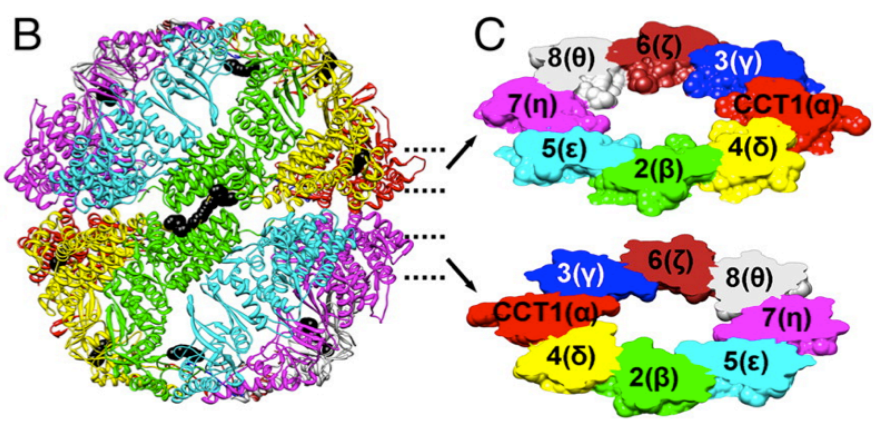

Kalisman N et al. PNAS 2012;109:2884-2889

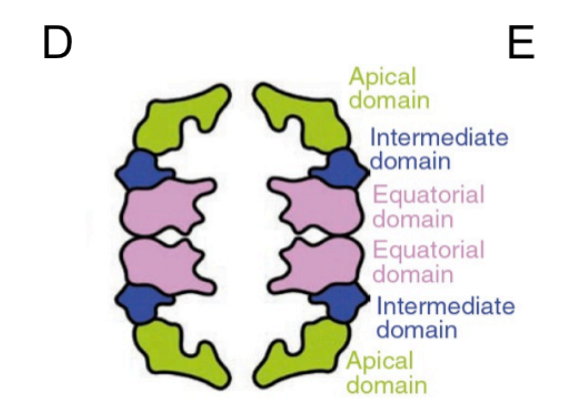

Muños et. al. PNAS 2011; 18

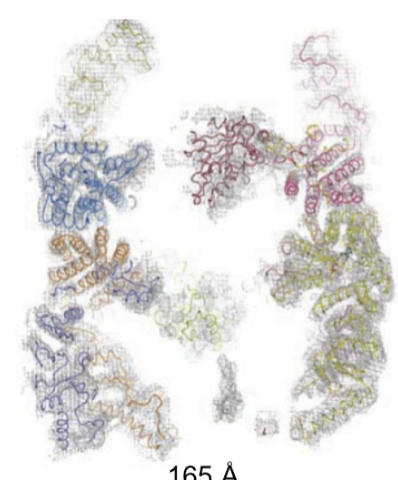

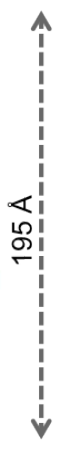




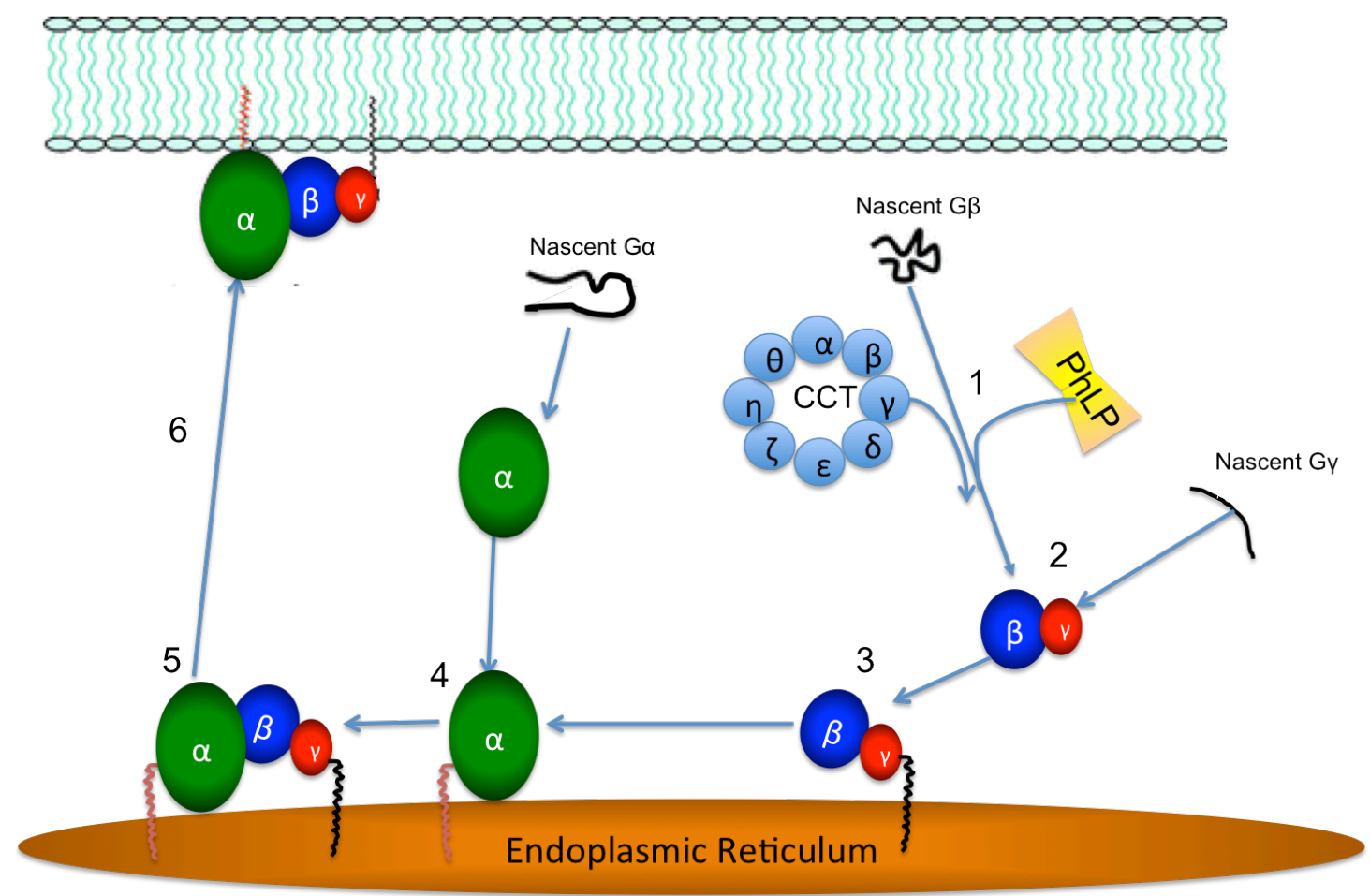

Figure 2. A model of $\mathrm{G} \beta$ biogenesis and assembly into the heterotrimeric G-protein complex at the plasma membrane. 1. Nascent G $\beta$ is folded by CCT with the help of the phosducin-like protein (PhLP) co-chaperone (9, 10). 2. All G $\beta$ subunits irreversibly associate with G $\gamma$ soon after biogenesis and exist as dimers, with the exception of the G $\beta 5$ subunit. It is not clear when, but at some point PhLP dissociates and the G $\beta \gamma$ dimer is formed. 3. G $\gamma$ becomes farnesylated or geranylgeranylated, which anchors $G \beta \gamma$ to the membrane, and is then targeted to the cytoplasmic surface of the endoplasmic reticulum (ER). 4 . Details of $\mathrm{G} \alpha$ biogenesis are still unclear, but it becomes either palmitoylated or myristoylated to anchor it to the membrane. 5 . It is speculated that $G \alpha$ associates with $G \beta \gamma$ at the ER and 6. the heterotrimeric G-protein complex is subsequently trafficked to the plasma membrane (11).

G-proteins are a highly conserved group of molecules involved in a great number of signaling processes. They are composed of $\alpha-, \beta-$, and $\gamma$-subunits, which are bound together in the inactive form. In this heterotrimeric state, guanosine di-phosphate (GDP) is bound to the $\mathrm{G} \alpha$-subunit $(\mathrm{G} \alpha)$. Upon activation, the $\mathrm{G} \alpha$ exchanges GDP for guanosine triphosphate (GTP). This exchange triggers $G \beta \gamma$ to dissociate from $\mathrm{G} \alpha$ and both $\mathrm{G} \alpha$-GTP and $\mathrm{G} \beta \gamma$ are then free to activate downstream effectors. While much is known about the signal transduction, far less is known about the biogenesis and assembly of G-proteins. Currently it is known that these proteins come from several distinct genes. In humans, 16 genes code for $\mathrm{G} \alpha, 12$ genes code for $\mathrm{G} \gamma$, and 5 genes code for $\mathrm{G} \beta$. Figure 2 shows a model of $\mathrm{G} \beta$ biogenesis and subsequent assembly into the heterotrimeric G-protein complex at the plasma membrane. CCT folds nascent G $\beta$ with the help of the essential cofactor, phosducin-like protein (PhLP), but the order of these steps are not well defined.

PhLP a member of the phosducin (Pdc) family of proteins. The Pdc gene family is divided into three subgroups. Subgroup I consists of Pdc and PhLP1, which are both able to bind G $\beta \gamma$ subunits with high affinity (12). PhLP1 is ubiquitously expressed (13) and is considered to be a modulator of G $\beta \gamma$ function (14) through its interaction with CCT. In contrast, Pdc expression is restricted to retinal photoreceptor cells and the pineal gland (10). Subgroup II consists of PhLP2A and PhLP2B, which were both discovered in humans, (15). In yeast, the PhLP2 ortholog does not bind G $\beta \gamma$, but is essential for cell growth in both the yeast Saccharomyces cerevisiae (16) and the soil amoebae Dictyostelium discoideum (15). Subgroup III is comprised of only PhLP3 (15), which is thought to participate in $\beta$-tubulin and possibly actin folding (17), but binds G $\beta \gamma$ poorly (16). While the members of the Pdc gene family have seemingly unrelated functions, one commonality among PhLP 1-3 is that they are all thought to participate in protein folding by acting as co-chaperones (12). 
Figure 3: Formation of the CCT-PhLP complex
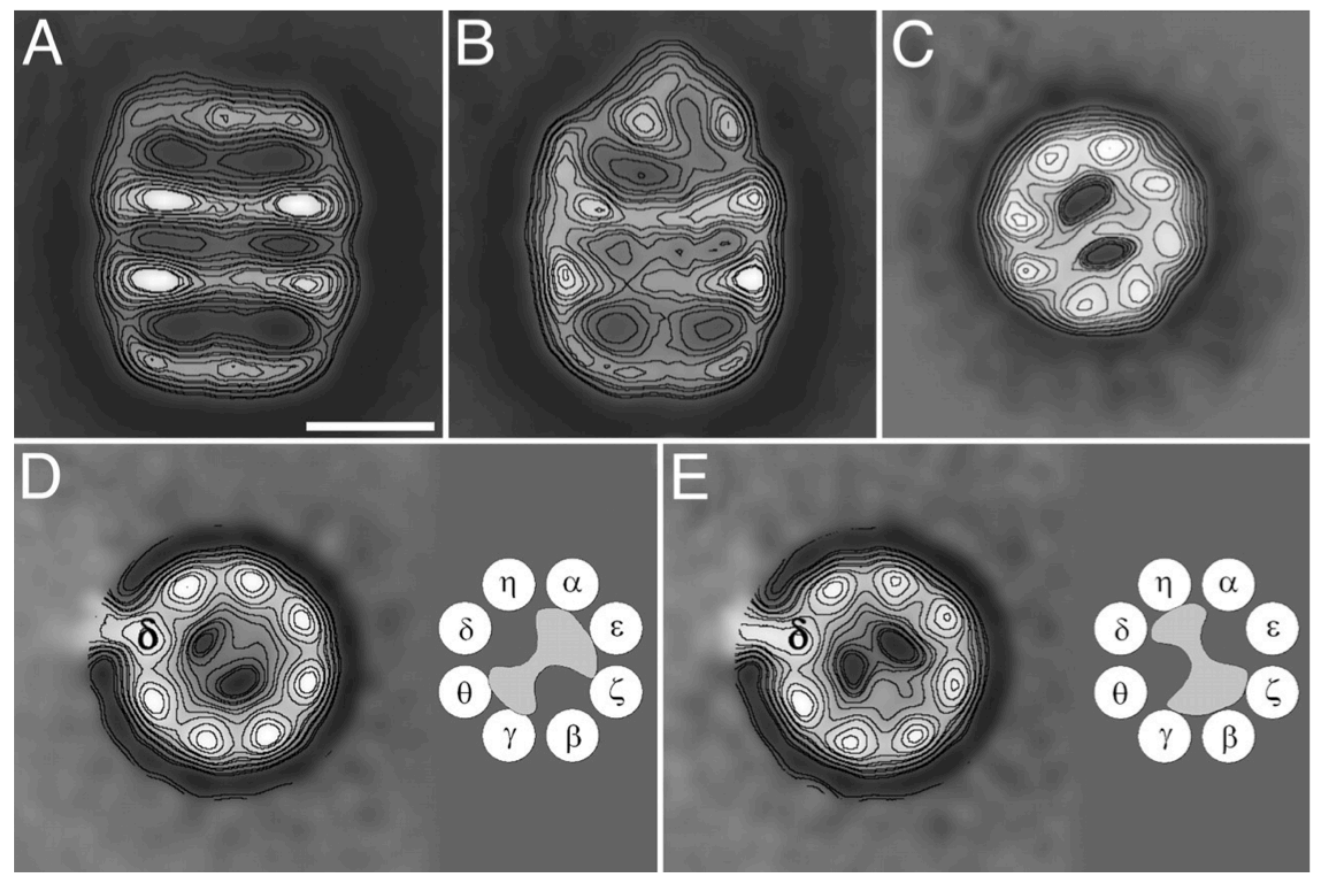

Two-dimensional average images of negatively stained CCT:PhLP complex. (A) Average image of side views obtained from 243 CCT particles of apo-CCT. (B) Average image obtained from 286 side views of CCT:PhLP complexes. (C) Average image obtained from 4,225 top views of CCT:PhLP complexes. ( $D$ and $E$ ) Average images of the two types of top views of CCT:PhLP:8g (anti-CCT) immunocomplexes (average of 324 and 626 particles). The subunit labeled by the antibody is marked with "." (Scale bar, $100 \AA$.) A schematic model of the each mode of PhLP binding, with the topology of the CCT subunits according to ref. 24, accompanies each average image. (Martín-Benito $\mathrm{J}$ et al. PNAS 2004;101:17410-17415)

PhLP1 is the most well studied studied of this family. Cryogenic Electron Microscopy (Cryo-EM) studies have shown that PhLP is not a substrate of CCT. Instead, PhLP interacts with CCT's apical domains on the top of the complex without any part of its mass entering the folding cavity (Figure 3). These studies have also shown that PhLP can interact with CCT in a one to one ratio and can interact with any of CCT's subunits to assume one of two conformations (18).

Although there has been extensive research into G-protein signaling, there are still many gaps in our understanding of G-protein biosynthesis. Because there are currently there are no available structures of this complex, nor is there a single published structure of PhLP I have used a homology modeling approach to generate the first known 3D structure of PhLP. The information that is gained from these structure-based studies can be combined with what is known from biochemical studies in order to gain isight into the interaction between PhLP and G $\beta$. Using this information I can make predictions to refine the mechanism of $G \beta$ biosynthesis. 


\section{CHAPTER 1: THE ROLE OF CCT IN G-PROTEIN ASSEMBLY}

\section{Introduction}

Initially most of the G-protein research had focused on $\mathrm{G} \alpha$, leaving $\mathrm{G} \beta \gamma$ largely ignored. However, more recent research has indicated that because of G $\beta^{\prime}$ s unique structure, it is able to interact with a wide variety of other proteins and plays a very important role in cell signaling. Structurally, G $\beta$ is a circular molecule composed of seven $\beta$-propeller WD40 repeats and belongs to the large family of WD40 proteins (23). Like other WD40 proteins, G $\beta \gamma$ is able to interact with a wide variety of proteins. X-ray crystallography of $G \beta$ co-crystalized with its binding partners has revealed that all of $G \beta^{\prime} s$ binding partners share a common interface (Figure 4). A recent review conducted on the structural interaction of G $\beta \gamma$ with its binding partners suggests that each protein interacts with $G \beta \gamma$ through a unique combination of residues within the common binding surface (24). This region of multi-target recognition called a "hot spot", is a region on the protein surface that is structurally flexible enough to accommodate multiple modes of binding (24). This interface is also the point of interaction between $\mathrm{G} \alpha$ and $\mathrm{G} \beta \gamma$ (figure 4D).

Heterotrimeric G-protein signaling begins with dissociation of $\mathrm{G} \alpha$-GTP from the $\mathrm{G} \beta \gamma$ dimer. While $\mathrm{G} \alpha$ subunit signaling is terminated by hydrolysis of GTP, G $\beta \gamma$ signaling must be terminated by re-association with $\mathrm{G} \alpha$ subunits or in a way that blocks the binding surface of G $\beta$. The re-association of the G-protein $\alpha-, \beta-$, and $\gamma$-subunits into the inactive heterotrimeric G-protein complex is a critical point in the regulation of overall G-protein signaling activity.

Phosducin-like protein (PhLP) plays an important role in $\mathrm{G} \beta$ biosynthesis, but the mechanism is not well understood. Our current understanding of this process was summarized by Willardson and Howlett in 2007 (Figure 5) (12). As a nascent $G \beta$ emerges, it forms a ternary complex by entering into the folding cavity of CCT and binding to PhLP and. It is not clear whether G $\beta$ associates with PhLP before or after entering CCT, but the interaction is controlled by PhLP because over-expression

Figure 4. G $\beta \gamma$ Crystal Structure

A. Schematic of $\mathrm{G} \beta_{1}$ showing the 7 WD40 repeats (Kobuta et al., 2006), B. $G \beta_{1} \gamma_{1}$ (Sondek et al., 1996), C. G $\beta_{1} \gamma_{1}$-phosducin (Gaudet et al., 1996) and D. $G \alpha_{t / \beta} \beta_{1} \gamma_{1}$ heterotrimer (Lambright et al., 1996) with $G \beta$ subunits are shown in light blue and $\mathrm{G} \gamma$ subunits in green

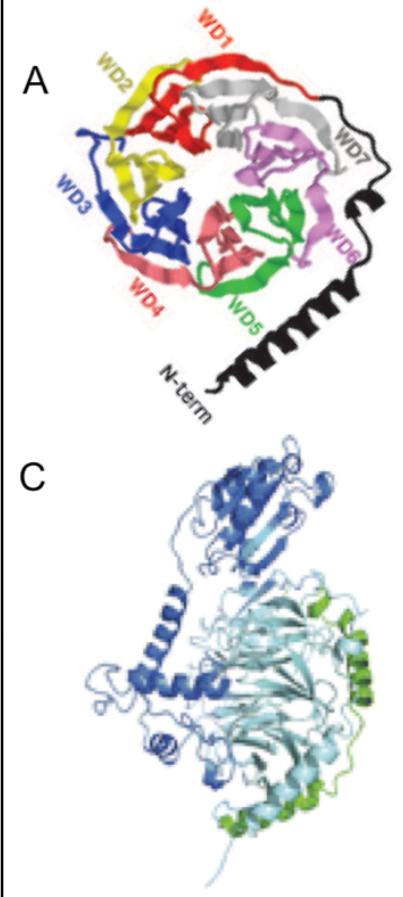

B

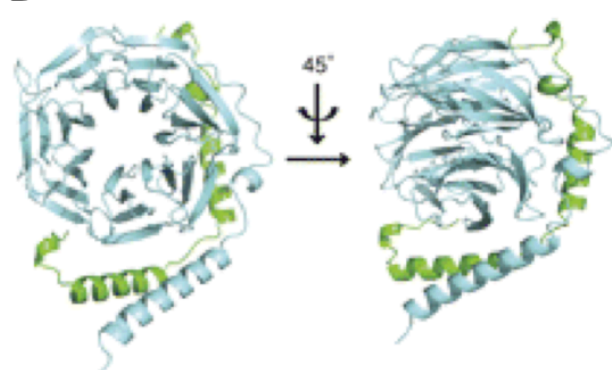

D

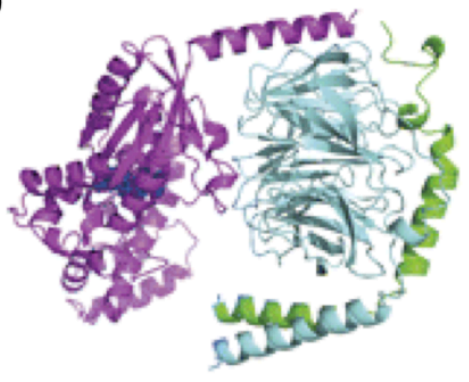
of PhLP decreases the binding of G $\beta$ to CCT (25). In this model it is theorized that the purpose of PhLP is to pull the folded G $\beta$ out of CCT and direct it to the membrane where it can associate with G $\alpha$. It is also thought that this mechanism is regulated by phosphorylation of PhLP. Phosphorylation of residues S18-20 by casein kinase 2 (CK2) is predicted to destabilize the ternary complex so that PhLP and the folded G $\beta$ can be released (25).

Experiments involving the truncated splice variant of phosducin-like protein (PhLP), referred to as PhLPSHORT (PhLPs) (19), have provided additional information that seems to support this mechanism. Despite having a significantly reduced affinity for $G \beta(14,21)$, PhLPs is still able to inhibit CCT in a dominant negative fashion $(20,25)$,

Immunoprecipitation (IP) experiments utilizing transgenic mice expressing PhLPs-FLAG (Tg+) in photoreceptors indicate that PhLPs is still able to interact with CCT (Figure 7A). The additional presence of the transducin $\beta$-subunit $\left(\mathrm{G} \beta_{1}\right)$ in the IP suggests that the trimeric PhLP(s)-CCT-G $\beta_{1}$ complex forms but cannot separate, leaving G $\beta$ trapped inside CCT (Figure 7B). This supports Willardson's model because the N-terminal residues that PhLPs lacks contain the residues implicated in CK2 phosphorylation as well as G $\beta$ binding. However, there are still many details of this mechanism that are unclear. 


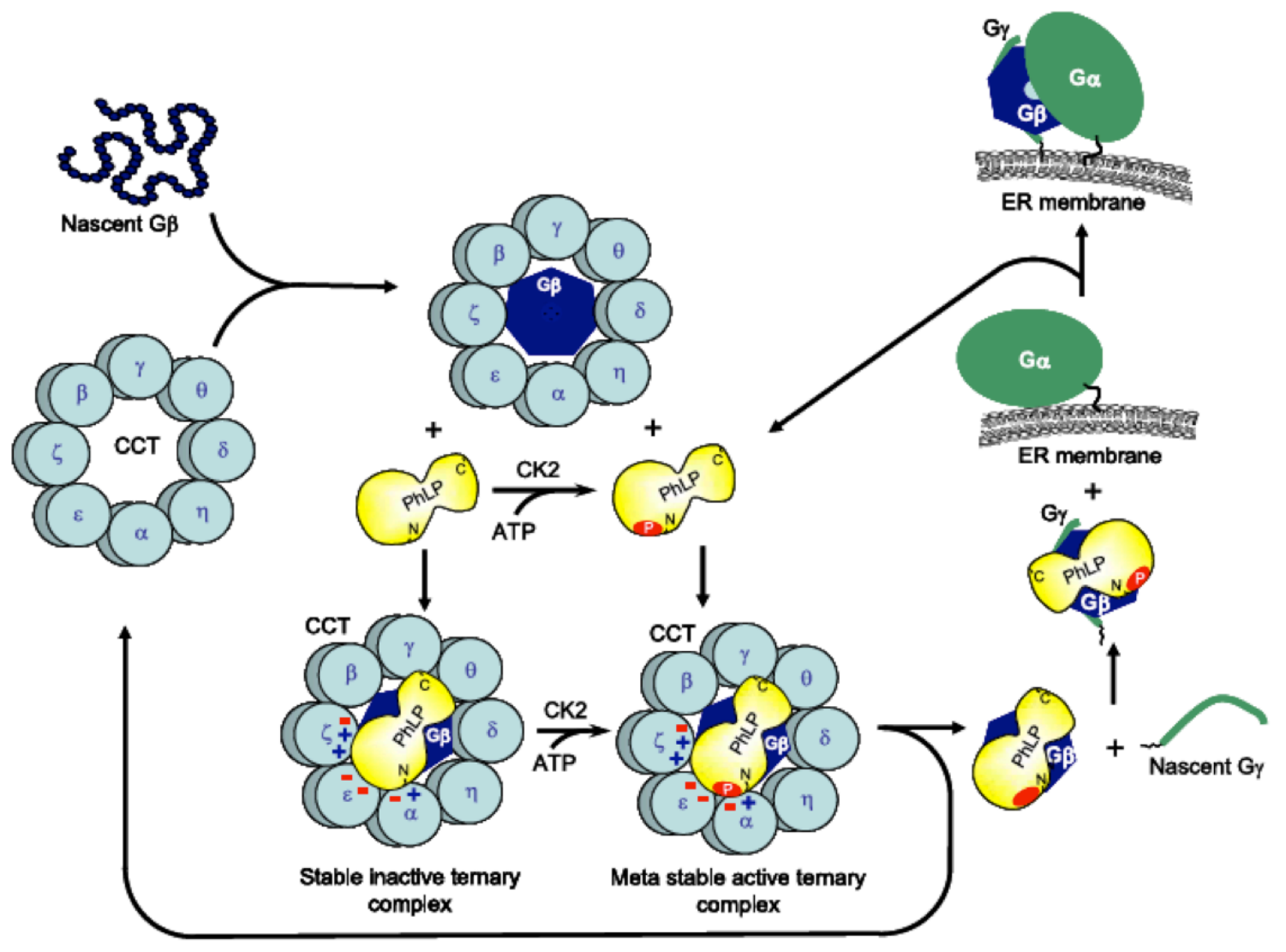

Figure 5. A mechanism describing the role of PhLP1 in G $\beta y$ dimer formation is depicted. Nascent G $\beta$ binds CCTwithin the folding cavity and PhLP1 associates above the G $\beta$, forming a ternary complex. If PhLP1 is not phosphorylated within the S18-20 sequence by CK2, the ternary complex is stable and inactive in G $\beta y$ assembly. If PhLP1 is phosphorylated at this site, then a PhLP1-G $\beta$ complex is released from CCT and interacts with Gy bound to DRiP78, forming the Gßy dimer. PhLP1 is released when $G \beta y$ associates with $G \alpha$ and the endoplasmic reticulum (ER) membrane, and the $G$ protein heterotrimer is then trafficked to the plasma membrane. PhLP is then free to catalyze another round of GßY assembly. (Willardson \& Howlett 2007)

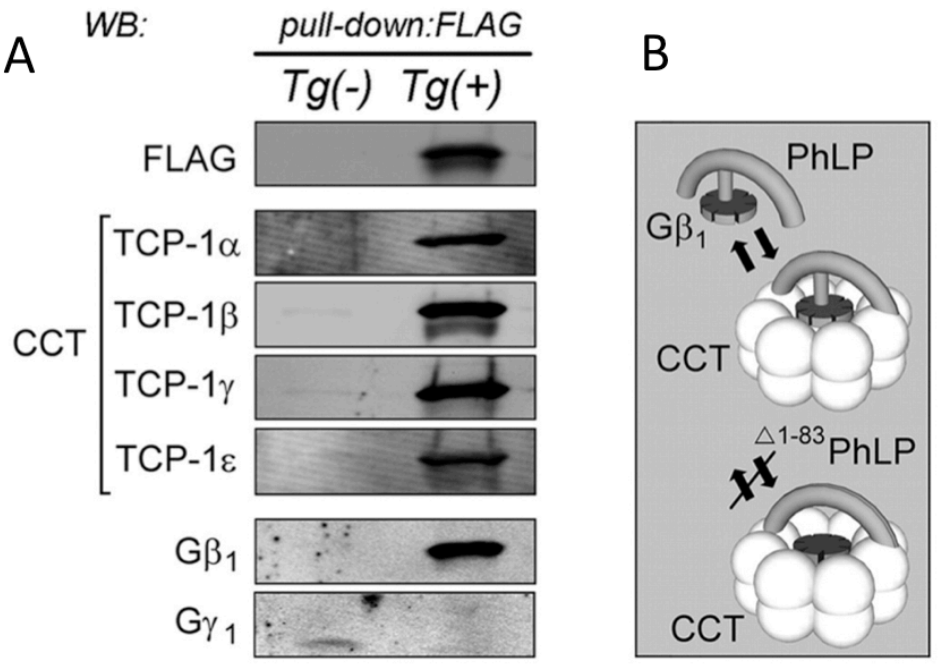

Figure 6. PhLPs, a truncated isoform of PhLP lacking the first 83 amino acids, is still able to form a ternary complex with CCT and GB. A. Western blot analyses of FLAG-agarose pull-down products in the retinal extracts of transgene-negative $\mathrm{Tg}$ $(-)$ and transgene-positive $\mathrm{Tg}(+)$ PhLPs mice at $\mathrm{P} 10$, detected with antibodies against FLAG, TCP-1 $\alpha$, TCP-1 $\beta$, TCP-1 $\gamma$, $\mathrm{TCP}-1 \varepsilon, \mathrm{G} \beta 1$, and $\mathrm{G} \gamma 1$, as indicated. $\mathrm{B}$, Model of PhLP-dependent delivery and release of G $\beta 1$ from the chaperonin CCT that shows formation of a stable complex between PhLPs ( $\triangle 1-83 P h L P), C C T$, and Gß1. (Posokhova E et al. 2011) 
In order to gain a better understanding of the involvement of PhLP in G $\beta$ biosynthesis, I combined experimental data with structural information. Using homology modeling, I was able to construct a 3-dimensional model of PhLP based on the crystal structure of the closely related protein phosducin (Pdc). Then I docked the PhLP model to the crystal structure of $G \beta 1$ to simulate binding. By identifying the residues involved in binding to $G \beta$, binding to CCT, and phosphorylation I demonstrate how PhLP is able to associate with both CCT and G $\beta$, and predict how phosphorylation of PhLP triggers the release of G $\beta$. Ultimately, this information may determine CCT's role in G-protein biosynthesis through its interaction with PhLP and G $\beta$.

\section{Results}

\section{Generating a 3D structure of PhLP}

In order to gain a better understanding of the role that CCT plays in G-protein biosynthesis, we must first figure out how the interaction between its co-chaperone, PhLP, and its substrate, G $\beta$. There are currently no published structures of PhLP. In order to determine how PhLP is interacting with G $\beta$, and to predict the mechanism of release I generated a 3D structure of PhLP based on homology modeling. Homology modeling is the most accurate and widely used method of protein structure prediction. It is based on the empirical observation that 3D structure is the most robustly conserved feature of homologous proteins. Even when the primary sequence has been altered by mutation, deletion, or insertions, the 3D structure of evolutionarily related proteins is largely preserved. Building a 3D model of an unknown "target" protein based on its primary amino acid sequence requires a closely related protein with a known 3D structure to act as a "template". There are different approaches to homology modeling, but they all follow four basic steps; 1 ) identify one or more proteins to serve as the 3D template, 2) sequence-structure alignment to line up amino acid residues of the target to the corresponding ones in the template, 3) use the sequence-structure alignment to generate a 3D model of the target protein, and 4) evaluate the model for correctness (Figure 7).

The phosducin family of proteins is very small and the proteins are evolutionarily conserved from simple single cell eukaryotes to higher order mammals. Fortunately, the crystal structure of rat phosducin (Pdc) has been solved (26). Phosducin and PhLP are both members of Subgroup II of the phosducin family, and both proteins have a high degree of similarity. To confirm that Pdc was the best protein to use as a template for mouse PhLP, the Position-Specific Iterative Basic Local Alignment Search Tool (PSIBLAST) was used via the Internet-based PredictProtein (www.predictprotein.org) website. The sequence of mouse PhLP1 has 301 amino acids and rat Pdc has 246 amino acids. They share $65 \%$ similarity in primary amino acid sequence with 109 identical and 88 similar residues, making it the best template with a crystal structure. Two different 3D structures of rat Pdc were available through the Protein Data Bank (PDB = 1B9X, and 2TRC), and both were used as template molecules for homology modeling.

Sequence alignment and the generation of a 3D model of PhLP1 were accomplished using INSIGHTII, a graphical molecular modeling program developed by Accelrys Inc. (formerly a product of MSI and BIOSYM).

\section{Figure 7. Basic homology modeling workflow}

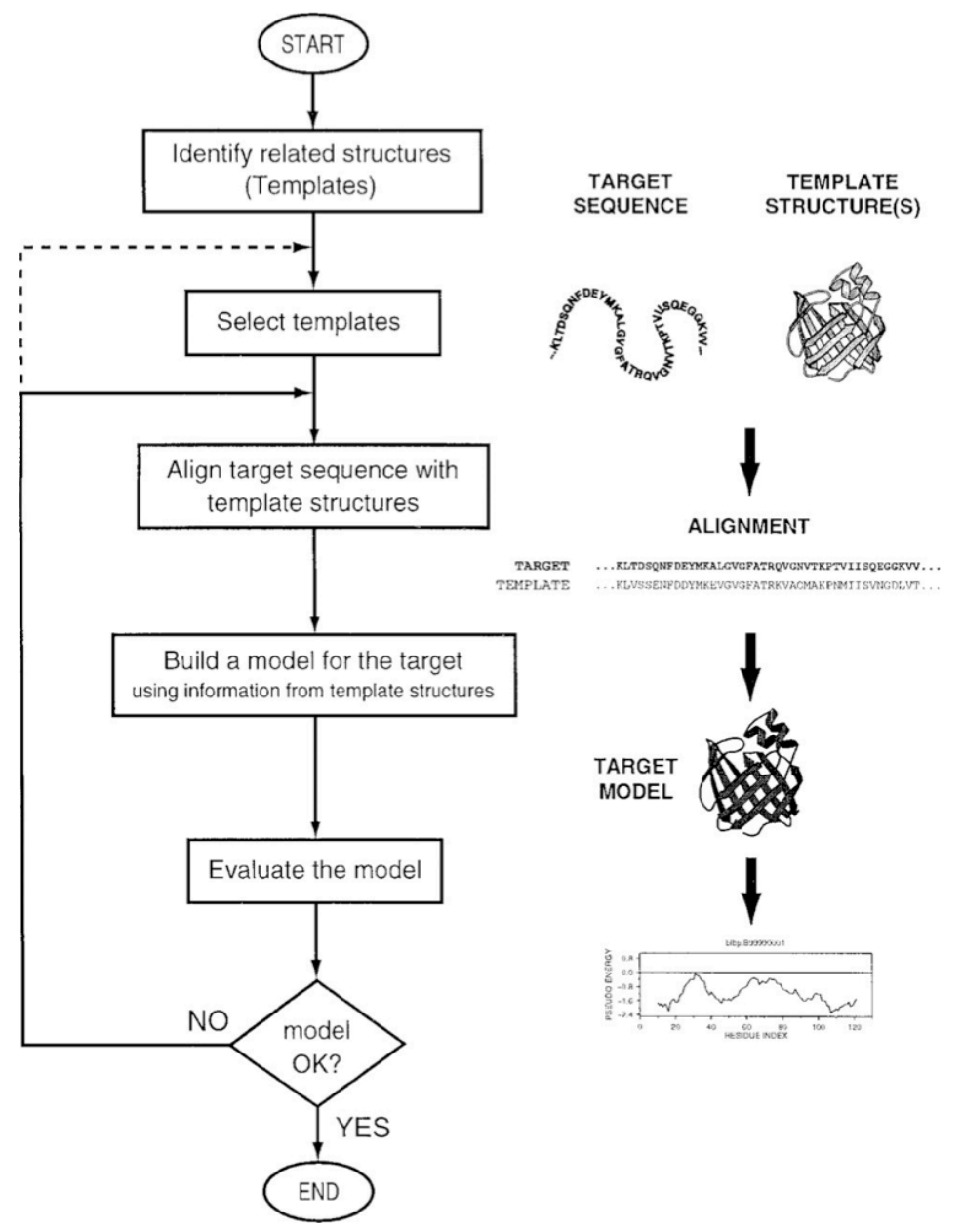


Using INSIGHTII, the amino acid sequence of PhLP was aligned to those of the two Pdc templates based coordinates obtained from their crystal structures. In order to confirm the sequence alignment and to determine how similar the structure of PhLP will be to Pdc, PredictProtein was used to predict the secondary structure, solvent accessibility and membrane helices. PredictProtein makes these predictions by combining results of the PHD and PROF servers in order to visually represent the likelihood of beta sheet or alpha helical secondary structures, disordered regions, and solvent accessibility occurring along the primary amino acid sequence (Figure 8). Secondary structure prediction was done for PhLP and Pdc individually and then compared back to the sequence-structure alignment generated by INSIGHTII (Figure 10). PredicProtein accurately predicted the secondary structural features of Pdc. Based on these prediction, the Cterminal domain of PhLP is very similar to Pdc but there were two notable differences in the N-terminus. First, in PhLP Helix 2 is predicted to be closer to Helix 1 while in Pdc Helix 2 is adjacent to Helix 3 . Secondly, the region of the Nterminus before Helix 1 is much longer in PhLP and is predicted to be disordered and solvent accessible. 

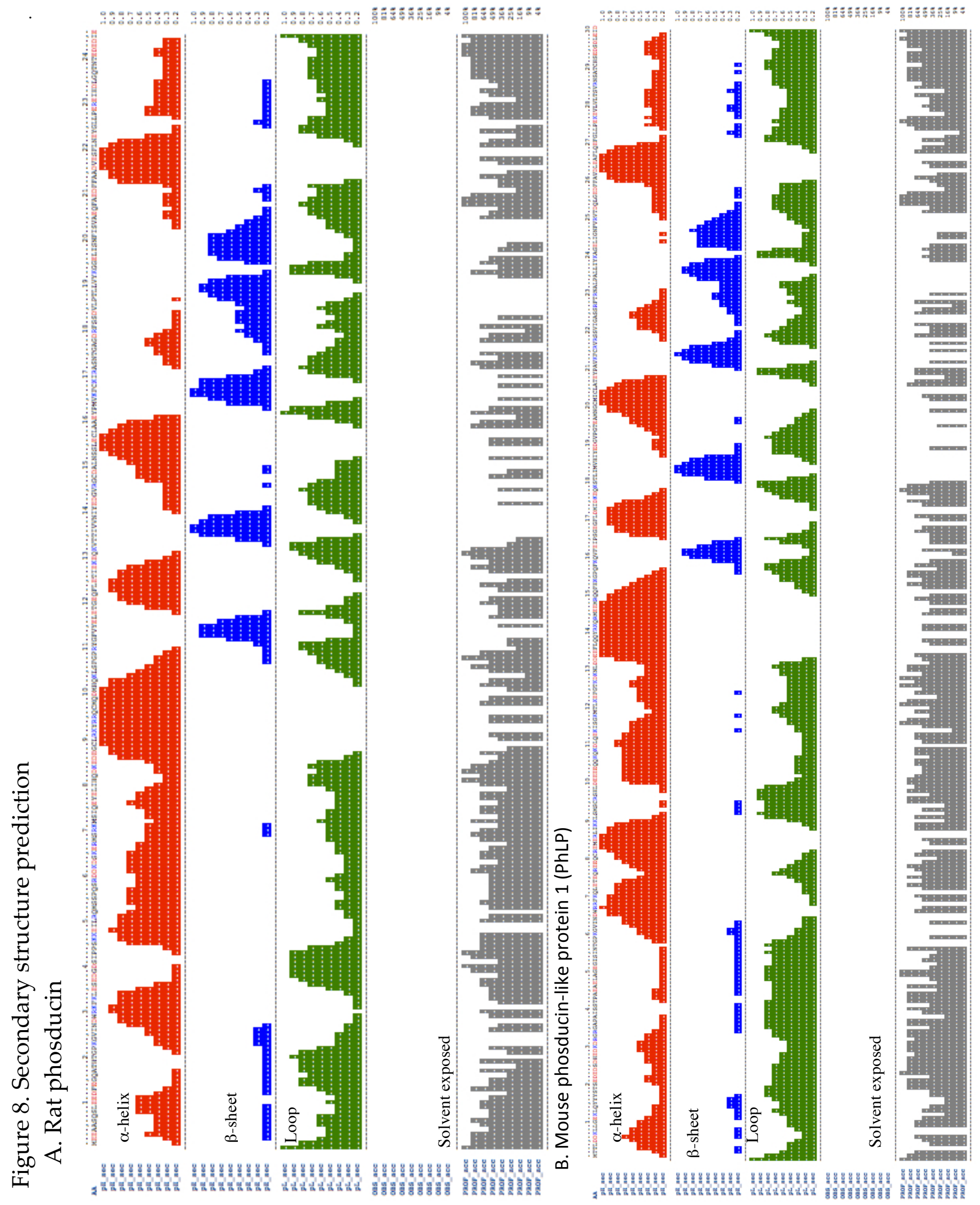
Figure 9. Sequence alignment between phosducin-like protein (PhLP1) and two template molecules (Phos1 and Phos2)

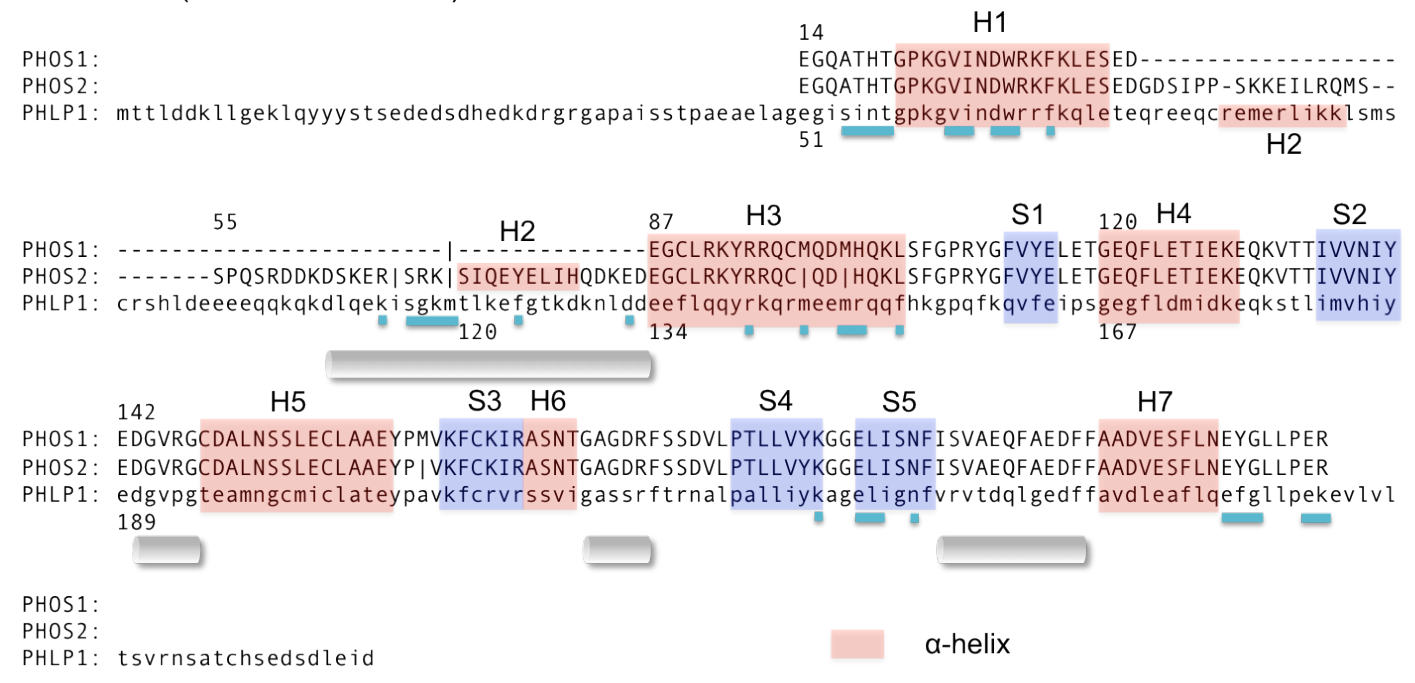

B-sheet

Residues on PhLP that bind CCT

Conserved residues that bind $\mathrm{G} \beta$

Generation of a 3D PhLP model was accomplished using the CONSENSUS module of INSIGHTII, using distance constraints derived from the reference protein structures (Figure 11). Superimposing the PhLP model onto the Pdc structure indicates the high degree of similarity between the two structures. In figure 11A Pdc is modeled by a light blue ribbon with the flexible $\mathrm{N}$-terminus and the $\mathrm{C}$-terminal thioredoxin domain indicated. The $\mathrm{C}$-terminus of Pdc is rigid, but the N-terminus, which is composed of $3 \alpha$-helices $(\mathrm{H} 1, \mathrm{H} 2, \mathrm{H} 3)$ is very flexible. Figure $11 \mathrm{~B} \& \mathrm{C}$ shows Pdc modeled by a dark blue ribbon and PhLP modeled by a green ribbon. The C-terminal thioredoxin domain is almost perfectly superimposed but, with the exception of $\mathrm{H} 3$, the N-terminal domain shows significant differences. Figure 11D shows the structure of PhLP in green with residues involved in G $\beta$ binding predicted by homology to Pdc shown in light blue, residues involved in CCT binding in white, and phosphorylation sites in red. The $\mathrm{N}$ - and $\mathrm{C}$-terminus are longer than that of Pdc, and are shown in figure $11 \mathrm{C} \& \mathrm{D}$ to extend out away from the rest of the molecule. Figure $11 \mathrm{C} \& \mathrm{D}$ suggests that portions of the N-terminus of PhLP are less disordered and exposed to solvent, but no conclusions can be drawn since there is no template for structure prediction. The red arrows in figure 11B\&C point to the region on helix-1 that is critical for G $\beta$ binding. These residues are conserved between PhLP and Pdc but since figure 11B indicates that they are not superimposed, it is likely that there are differences in G $\beta$ binding. The white arrows in figure 11B,C, and D point to a flexible loop that may be implicated in triggering the release of $G \beta$. The residues on PhLP that are involved in binding to CCT are shown in white in figure 11D. The flexible loop on the N-terminus of PhLP that is involved in CCT binding is not present in Pdc, which explains why phosducin is unable to bind CCT. Note that in Figure 11A only one $\alpha$-helix connects the $\mathrm{N}$-terminal domain of Pdc to the $\mathrm{C}$-terminal domain, while in figure 11C PhLP has two connections (pink arrows). $\mathrm{H} 3$ connects the $\mathrm{N}$ - and C-terminal domains of both PhLP and Pdc. The second connection between the $\mathrm{N}$ - and $\mathrm{C}$-terminus of PhLP is formed by residues on the N-terminus prior to H1. This suggests that PhLP is much less flexible than Pdc.

In order to determine how PhLP might interact with G $\beta$, I compared my model to the crystal structure of the PdcG $\beta_{1} \gamma_{1}$ complex (PDB = 2TRC). Initially, PhLP simply replaces Pdc in the Pdc-G $\beta_{1} \gamma_{1}$ complex to highlight the differences between the two proteins with respect to $G \beta$ binding (Figure 12). G $\beta 1 \gamma_{1}$ is shown in yellow, Pdc is shown in light blue, and PhLP is shown in green. In Figure $12 \mathrm{A \& B}$, Pdc makes three points of contact with G $\beta_{1 \gamma 1}$. H1 is the most critical for binding, and $\mathrm{H} 3$ as well the residues on the $\mathrm{C}$-terminal domain help to stabilize the complex. In comparison, PhLP seems to wrap around $G \beta_{1} \gamma_{1}$. In figure 12D the residues 1-83 of PhLP are colored in brown to illustrate the residues that are absent in PhLPs. These structures clearly indicate that PhLP does not interact with G $\beta 1 \gamma_{1}$ in the same way as Pdc because the N-terminus is intertwined with $G \beta$. The red arrows in figures $12 A \& C$ indicate that the binding of G $\beta$ to H1 of Pdc and PhLP may differ, which may affect the overall structure. In figures $12 \mathrm{C} \& \mathrm{D}$ the residues of PhLP that interact with CCT are shown again in white, the residues that contact G $\beta$ are in light blue, and residues 1-83 that are removed in PhLPs are 
shown in brown. The white arrow in figure 12C\&D points to where the loop between $\mathrm{H} 2$ and $\mathrm{H} 3$ of PhLP overlaps with G $\gamma$. This may support the theory that PhLP interacts only with G $\beta$ and not the G $\beta \gamma$ dimer.

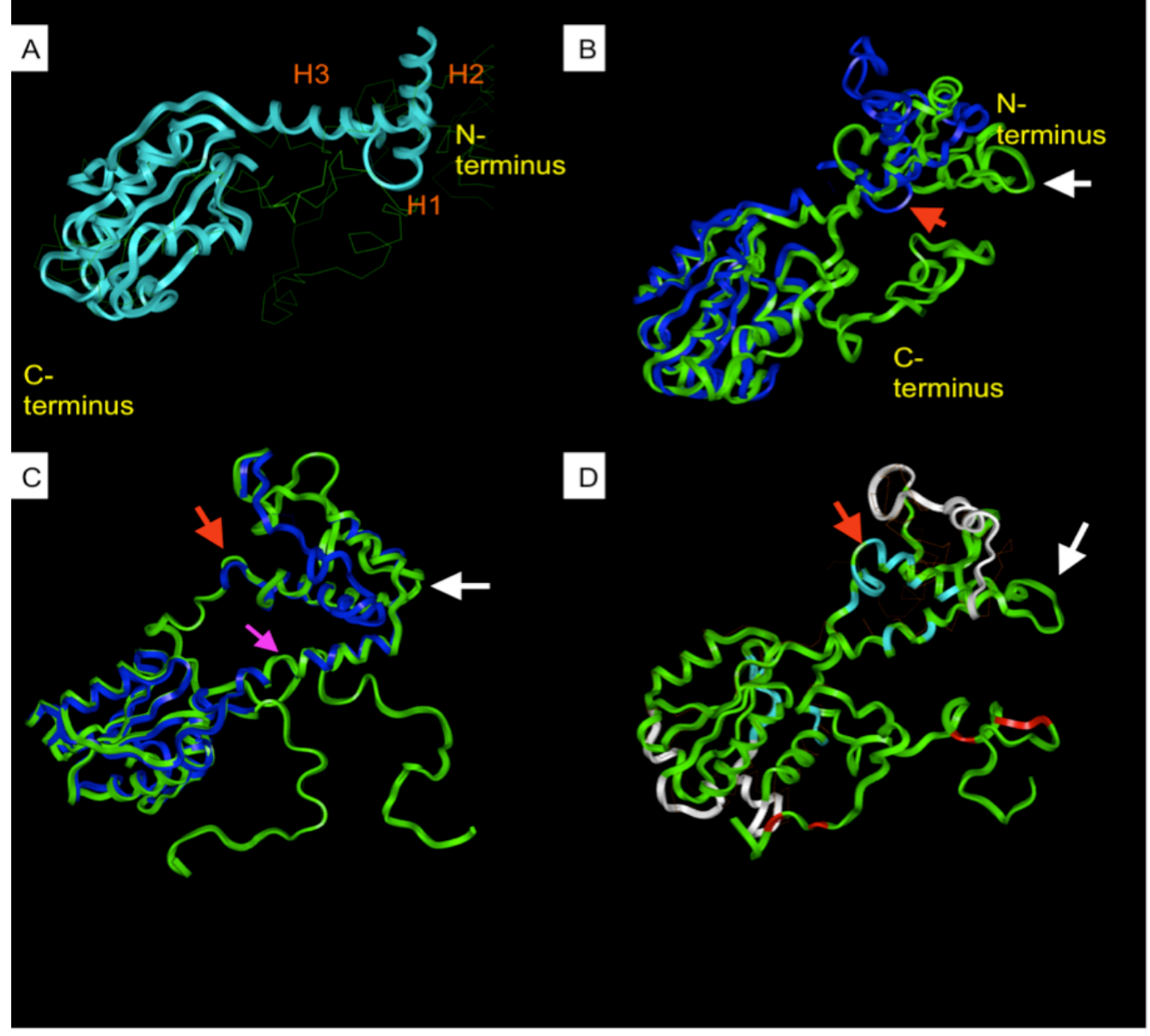

Figure 10. Generation of PhLP model and comparison to Phosducin (Pdc) template. A. Ribbon model of Pdc tempate. The three alpha helices in the $\mathrm{N}$ terminus are indicated by $\mathrm{H} 1, \mathrm{H} 2$, and $\mathrm{H} 3$. BD Ribbon model of PhLP (green) superimposed on Pdc (blue) shown at different angles. Red arrows indicate conserved residues on $\mathrm{H} 1$ that are critical for binding to $G \beta$. White arrows indicate the region that is thought to be involved in the release of $\mathrm{G} \beta$ from

PhLP or Pdc. D.

PhLP is shown alone. Residues involved in binding $G \beta$ are shown in light blue, those involved in binding CCT are shown in white, and phosphorylation sites are in red.
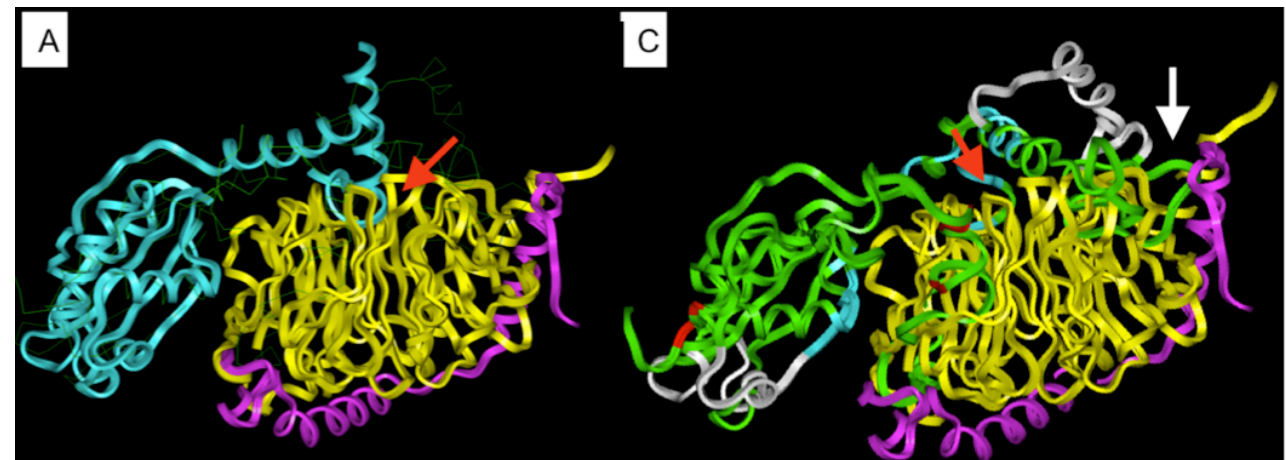

Figure 11. Comparison of $G \beta_{1} Y_{1}$ binding to phosducin (Pdc) (A\&B) to PhLP (C\&D) A-B. Ribbon model of Pdc tempate (light blue) binding to $\mathrm{G} \beta_{1}$ (yellow) in complex with $\mathrm{Gy}_{1}$ (pink). C-D.

PhLP (green)replaces $\mathrm{Pdc}$ in the complex shown in A\&B.

Residues involved in
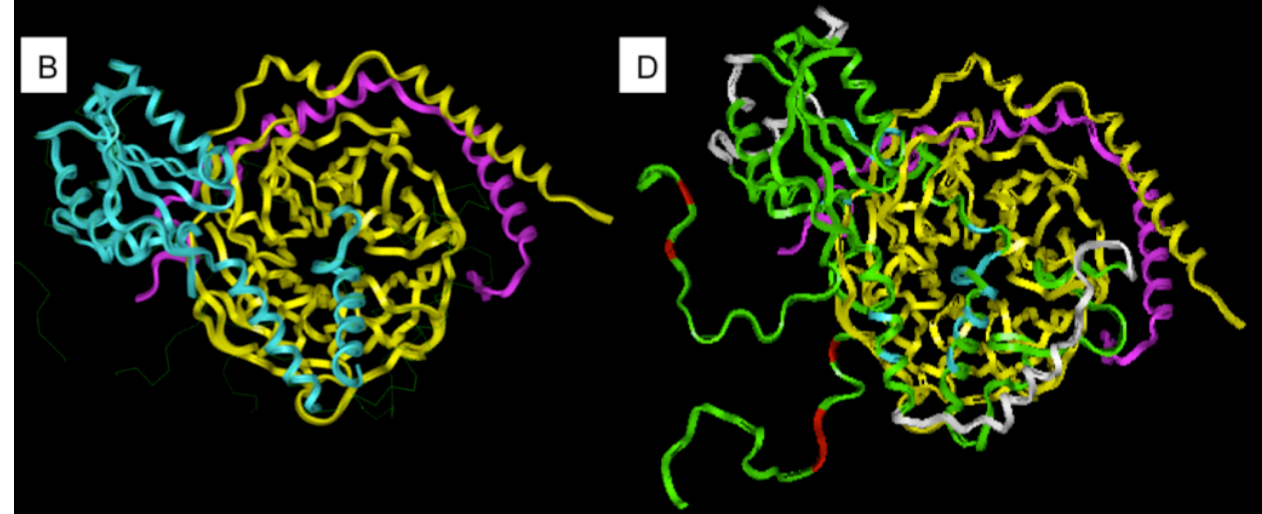
binding $G \beta$ are shown in light blue, those involved in binding CCT are shown in white, and phosphorylation sites are in red. Red arrows indicate conserved residues on $\mathrm{H} 1$ that are critical for binding to $G \beta$. White arrows indicate the region that is thought to be involved in the release of $\mathrm{G} \beta$ from PhLP or 


\section{Discussion}

Despite the similarity between phosducin and PhLP there are subtle differences in their structure. By combining the information that we have gathered from homology modeling with biochemical data we can speculate about PhLP's function in three different areas. First, these results help to explain how PhLP and PhLPs are both able to interact with CCT and G $\beta$ simultaneously. The 3D model that I generated of PhLP shows that this is possible because the binding sites are distinct. Furthermore, the mechanism of CCT inhibition by PhLPs that was proposed by our lab (Figure 6) is supported because neither the CCT nor the G $\beta$ binding sites are perturbed in PhLPs. Second, they reveal important information about the interaction between PhLP and G $\beta$ that is suggestive of its role in G-protein biosynthesis.

The mechanism of $\mathrm{G} \beta$ biosynthesis proposed initially proposed by Georgi Lukov and Barry Willardson in 2004 initially suggested that PhLP directly interacts will nascent G $\beta$ and this interaction is stabilized by CK2 phosphorylation of PhLP (22). This mechanism was later refined by Barry Willardson and Alyson Howlett in 2007 suggesting that nascent $\mathrm{G} \beta$ first binds to CCT, which then recruits PhLP (12)(Figure 4). In this later model, CK2 phosphorylation functions to release G $\beta$ from CCT. The PhLP-G $\beta$ complex then binds G $\gamma$ forming the PhLP-G $\beta \gamma$ complex. Finally, G $\alpha$ replaces PhLP to form the heterotrimeric G-protein complex at the membrane. The first issue that remains to be resolved in this model pertains to the purpose of PhLP. Is PhLP required for the folding of G $\beta$ by PhLP, to prevent G $\beta$ from being released from CCT before it is properly folded, to stabilize the folded G $\beta$ until it can be stabilized by $G \gamma$, or any combination of those roles? The second issue is that although the evidence of CK2 phosphorylation causing release of PhLP-G $\beta$ from CCT is well supported (25), the mechanism by which G $\beta$ is released by PhLP is still unclear.

Based on the 3D model that I generated of PhLP I have come up with a revised mechanism of PhLP's involvement in G-protein biosynthesis (Figure 12). 1. Nascent G $\beta$ enters the folding cavity after it is translated. PhLP does not require G $\beta$ to bind CCT (18). I believe that the first 50 amino acids in the long unstructured N-terminus of PhLP may help to weaken the interaction between PhLP and CCT. However, this is purely speculative because the structure of this region could not be determined. 2. Once G $\beta$ enters the inner chamber of CCT it becomes partially folded, and 3. PhLP will be able to bind with higher affinity because there are more points of contact. I predict that the long unstructured Nterminus of PhLP pulls G $\beta$ out of CCT after its folded. When PhLP binds to CCT and G $\beta$ to form the tertiary complex, this strand becomes wedged in between G $\beta$ and the C-terminal thioredoxin domain of PhLP, which has a net charge of 19. Furthermore, I predict that when H1 of PhLP binds to G $\beta$ it causes the N-terminal strand to rotate, exposing residues S18-20. 4. When these residues becomes phosphorylated by CK2 the charge repulsion causes PhLP's N-terminal strand to move away from the C-terminal domain, 6. effectively prying $\mathrm{G} \beta$ out of $C C T^{\prime}$ s inner chamber.

I have also been able to make a prediction as to how and when $G \beta$ is released from PhLP based on this model. Previous studies have shown that complexes of PhLP and G $\beta$ were found that do not contain $G \gamma$ (22), which supports PhLP's role in stabilizing G $\beta$ before $G \gamma$ can bind and form the stable G $\beta \gamma$ dimer. From the model of the PhLP-G $\beta_{1} \gamma_{1}$ complex shown in figure $12 C \& D$, it is clear that PhLP does not bind to G $\beta$ like Pdc. The N-terminus of Pdc is flexible and can orient the rest of the molecule around G $\beta \gamma$. In contrast, PhLP is more rigid and because of this I believe that PhLP and G $\gamma$ are mutually exclusive in their interaction with G $\beta$. I predict that the key to PhLP's release of G $\beta$ can be found in the loop between $\mathrm{H} 2$ and $\mathrm{H} 3$ indicated by the white arrows in figure 10 and figure 11. The region of $\mathrm{G} \beta$ that is critical for its interactions with binding partners is located on the top of the torus created by the $\beta$ propeller folds (24). I predict that in the absence of $G \gamma$, PhLP pinches the N-terminal $\alpha$-helix of G $\beta$ in toward the hydrophobic residues on the fifth $\beta$-propeller blade (WD5 in Figure 4A). This functions to stabilize G $\beta$ as well as hold it in a position to readily accept $G \gamma$. The interaction between $G \gamma$ and $G \beta$ is very strong. 7. Once $G \gamma$ comes in contact with $G \beta$ it will push the N-terminal $\alpha$-helix of G $\beta$ away from WD5, causing PhLP to loosen its hold on G $\beta$. 8. At this point PhLP's affinity for G $\beta$ will be so low that it will easily dissociate as $\mathrm{G} \gamma$ becomes either farnesylated or gernylgernylated at the endoplasmic reticulum. Lastly, 9. G $\beta \gamma$ associates with $\mathrm{G} \alpha$ and 10. the heterotrimeric complex is trafficked to the plasma membrane. 


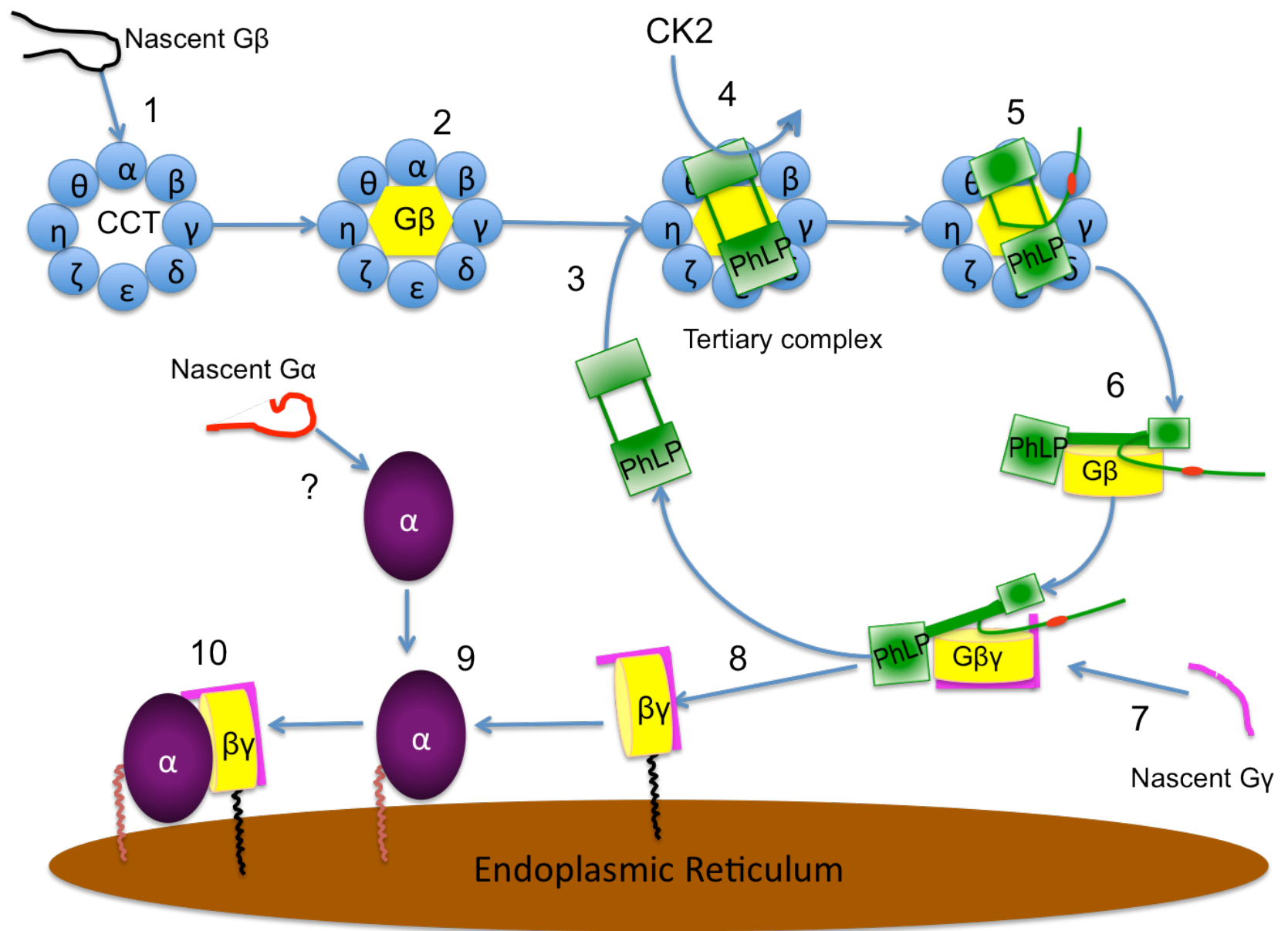

Figure 12. Revised model of $\mathrm{G} \beta$ biogenesis based on prediction from the 3D structure generated of PhLP. 1 . Nascent G $\beta$ enters the folding cavity after it is translated. 2. Once G $\beta$ enters the inner chamber of CCT it becomes partially folded, and 3. PhLP binds forming the tertiary complex and helps to complete the folding of $\mathrm{G} \beta$. When $\mathrm{H} 1$ of PhLP binds to G $\beta$ it also causes the N-terminal strand to rotate, exposing residues S18-20. 4. When these residues becomes phosphorylated by CK2 the charge repulsion causes PhLP's N-terminal strand to move away from the C-terminal domain, and 6. PhLP-G $\beta$ disassociates from CCT. 7. PhLP primes G $\beta$ for binding to $\mathrm{G} \gamma$. Once $\mathrm{G} \gamma$ binds, it displaces PhLP reducing its affinity for G $\beta$. 8. PhLP's easily dissociate as G $\gamma$ becomes either farnesylated or gernylgernylated at the endoplasmic reticulum 9. G $\beta \gamma$ associates with G $\alpha$ and 10. the heterotrimeric complex is trafficked to the plasma membrane. 


\section{ChAPTER 2: THE ROLE OF CCT IN THE REGULATION OF G-PROTEIN SIGNALING}

\section{Introduction}

PhLP is alternatively spliced, resulting in the loss of the first 83 amino acids (Figure 13). The truncated variant is referred to as PhLPнновт (PhLPs) (19), and while full length PhLP has ubiquitous expression, PhLPs is primarily found in the adrenal gland (19). This truncation removes amino acid residues that are critical for binding to G $\beta$. Because of this, PhLPs has a much lower affinity for G $\beta$ than PhLP (20) and binds G $\beta$ very poorly $(14,21)$. Despite the weak interaction with purified G $\beta$, PhLPs can still interact with and inhibit CCT (10). However, this interaction has also been shown to inhibit $G \beta \gamma$ signaling and protein expression in a dominant negative fashion $(20,22)$. Because PhLPs is such a potent inhibitor of CCT, is commonly used as a tool in the lab to study CCT activity. In cells, PhLPs expression additionally causes inhibition of $G \beta \gamma$ signaling and a profound reduction of $G \beta \gamma$ protein expression $(20,22)$. Since PhLPs does not interact with G $\beta$ directly, the effects on G-protein expression are thought to be an indirect consequence of CCT inhibition (10). In order to study the effect of CCT inhibition on G-protein signaling in vivo, our lab created several lines of mice that constitutively expressed PhLPs in rod photoreceptors. Photoreceptors in the eye are classically used for G-protein research due to the uniquely high concentration of a single type of G-protein referred to as transducin. Transducin is a heterotrimeric G-protein composed of transducin- $\alpha\left(\mathrm{Gt}_{\mathrm{t}} \alpha_{1}\right)$, transducin- $\beta\left(\mathrm{G} \beta_{1}\right)$, and transducing- $\gamma\left(\mathrm{G} \gamma_{1}\right)$ subunits. While $\mathrm{G} \beta_{1}$ and $\mathrm{G} \gamma_{1}$ are common to both rod and cone photoreceptors, $\mathrm{G}_{t} \alpha_{1}$, is specific to rods. Because approximately $96 \%$ of mouse photoreceptors are rods, using rod photoreceptors allows us to conduct experiments in cells enriched in Gproteins.

Our lab had previously created three lines of transgenic mice that constitutively express PhLPs in order to study the effects of CCT inhibition in vivo. In these transgenic mice, expression of ${ }^{1-83} \mathrm{PhLP}$ (PhLPs) with a FLAG tag is under the control of a modified $4.4 \mathrm{~kb}$ rhodopsin promoter (Figure 14A). Beginning at age P5 in mice, the rhodopsin promoter becomes active and PhLPs-FLAG is expressed in the transgenic mice. The resulting mouse lines were confirmed by tail PCR genotyping methods to determine transgenic positive $(\operatorname{Tg}(+))$, and transgeneic negative $(\operatorname{Tg}(-))$ mice for each line. PhLPs protein expression levels were determined by immunoprecipitation (IP) with $\alpha$-FLAG antibody and western blotted using rabbit anti-FLAG antibodies (Figure 14B). The resulting transgenic mouse lines had varying levels of PhLPs expression. Line 1178 is the "high expressor", 1054 is the "middle expressor", and 1065 is the "low expressor" of PhLPs. The level of PhLPs protein expression corresponded to a progressive loss in photoreceptors as seen from hemotoxylin and eosin staining of retinas (Figure 14C) and a loss in nuclei (Figure 14D) with a severity directly correlated with the level of PhLPs expression.

Figure 13: PhLP is alternatively spliced resulting in "PhLP SHORT (PhLPs)

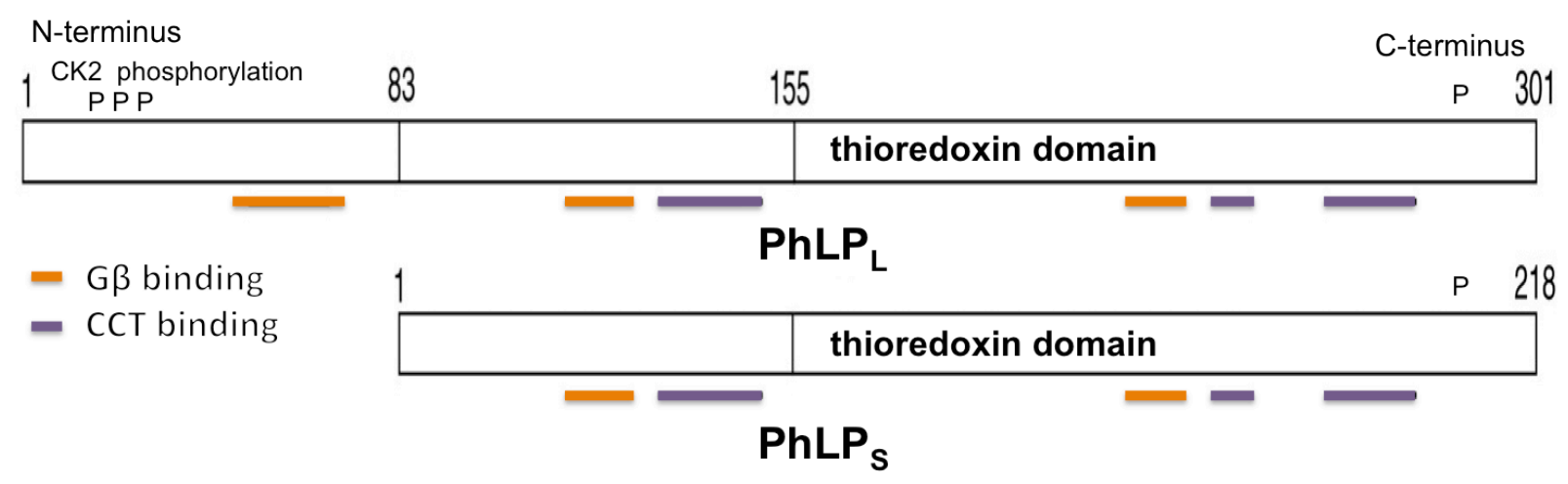

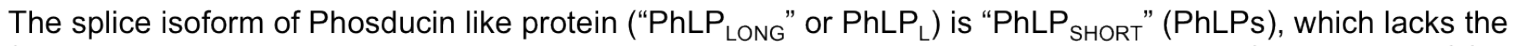
first 83 amino acids in. The region that is lost in PhLPs contains residues that are critical for binding to $G \beta$ as well as S18-20 phosphorylation sites that are thought to be involved in the release of G $\beta$ bound PhLP from CCT. 
Figure 14. Constitutive PhLPs expression in transgenic mice

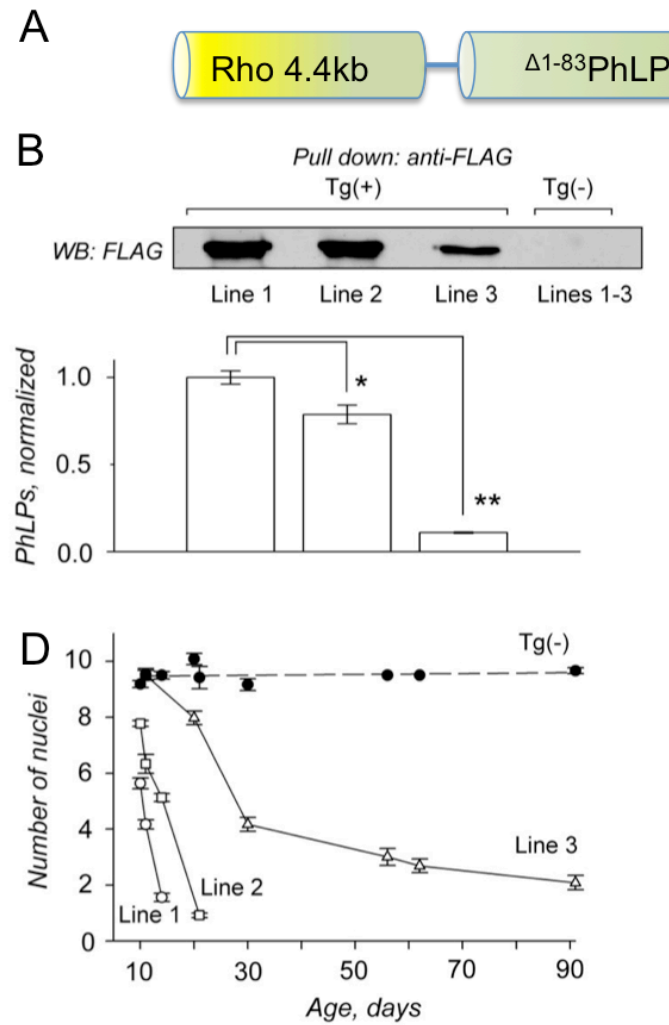

C

ONL [
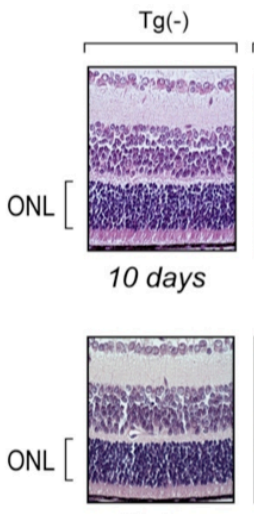

10 days

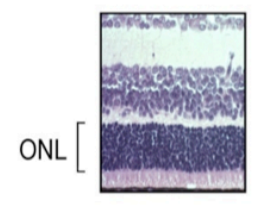

10 days

\section{FLAG}

S

PolyA

A. Transgene utilized for the expression of FLAG-tagged PhLPs $(\triangle 1-83 P h L P)$ in mouse photoreceptors. B. Immunoprecipitation of whole retina extracts from 3 different lines of transgenic mice expressing PhLPs at post-natal day 8 . Protein levels were normalized to line 1, the high expressor in order to compare expression of PhLPs between the lines. C. Hemotoxylin and Eosin staining of retinas taken from transgenic positive mice $(\mathrm{Tg}(+))$ at different ages from each line compared to transgenic negative controls $(\operatorname{Tg}(-))$ at post-natal day 10. D. Quantification of photoreceptor cells based on the number of nuclei.

Figure 15. PhLPs reduces expression of transducin subunit expression at the protein and mRNA level

A. $T g(-) T g(+)$

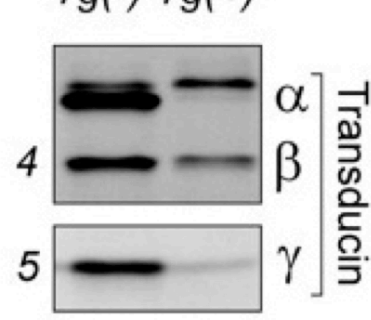

Posokhova et al., 2011

B.

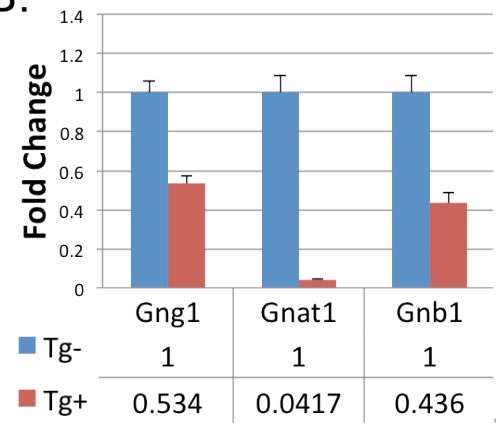

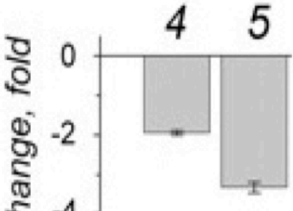

A. Measurement of the change in Transducin subunit expression levels in transgenic PhLPs mice. Left panel, representative Western blots showing the levels of the indicated proteins in the whole retina extracts of $\mathrm{Tg}(-)$ and $\mathrm{Tg}(+)$ littermates at P10 $(n=3)$. Right panel, change in transducin subunit protein levels by quantitative Western blotting, B. measurement of the change in gene expression level of transducin subunits from whole retina extracts of $\mathrm{Tg}(+)$ compared to $\mathrm{Tg}(-)$ littermates at P10 $(n=3)$ 
Western Blot analysis showed that PhLPs expression resulted in a reduction of $G \beta_{1}, G \gamma_{1}$, and Gt $\alpha_{1}$ (Figure 14A). The loss of G $\beta_{1}$ can be explained by the inhibition of CCT, which is required for folding nascent G $\beta$. Similarly, the loss in $\mathrm{G} \gamma_{1}$ is not surprising because without $\mathrm{G} \beta$ it will simply be degraded. However, the effect on $\mathrm{G}_{\mathrm{t}} \alpha_{1}$ was very unexpected. $\mathrm{G}_{t} \alpha_{1}$ protein levels were so low that the fold change could not be quantified. The band seen in the blot for $\mathrm{G}_{t} \alpha_{1}$ is nonspecific (Figure 14A). Further analysis revealed that gene expression levels showed a similar reduction. While gene expression of $\mathrm{G}_{1}(\mathrm{Gnb} 1)$ and $\mathrm{G} \gamma_{1}$ (Gng1) was reduced by roughly half, $\mathrm{G}_{\mathrm{t}} \alpha_{1}$ (Gnat1) mRNA had a greater than $95 \%$ reduction (Figure 14B). Currently there is no known mechanism to explain the loss of Gt $\alpha_{1}$ expression. These findings represent the first known evidence of connecting the activity of chaperonin CCT to G-protein gene activity.

The focus of this chapter is on gaining a better understand of CCT's role in the regulation of G-protein signaling. We used a microarray to show that the inhibition of CCT does not result in a wide-spread reduction in gene expression. Instead, only a very small subset of genes are significantly affected. Because the expression of PhLPs in the constitutive mouse lines cannot be controlled, our lab also began developing lines of transgenic mice that have inducible expression of PhLPs (iPhLPs). The iPhLPs mice will allow we to express PhLPs in mature photoreceptors in order to determine if the inhibition of CCT affects a developmental process. 


\section{Results}

\section{Microarray Analysis}

One possible explanation for the loss of $\mathrm{Gt}_{1} \alpha_{1}$ at the protein and mRNA level due to PhLPs expression is that inhibition of CCT leads to loss of a transcription factor. In order to test this, the Affymetrix GeneChip Whole Transcript Exon Array was used to monitor the changes in mRNA expression in transgenic mice expressing PhLPs $(\mathrm{Tg}(+))$ compared to littermate control mice $(\operatorname{Tg}(-))$. Exon Arrays are a high-density microarray platform with a total of $\sim 6.5$ million probes targeting all the annotated and predicted exons in the genome. A single gene on an Exon Array is assigned a set of 4 core probes targeted to each putative exonic region of a single gene, and collectively the probes representing the same gene are referred to as a "transcript cluster" (Figure 16A). Retina samples were collected from each of the three constitutive mouse lines, 1178, 1054, and 1065 at P10. For each line, three Tg+ mice were compared against Tg- littermate controls (Figure 16B). The results of each chip were first background subtracted using the signal from a set of 37,687 genomic and antigenomic background probes that are not matched to any putative transcript region. The data was then normalized using GeneBASE Probe Effects, which multiplies the data by a scaling factor so that the median intensity of all core probes in an array equals 100 (Figure 17).

Because some probes respond poorly to target signals, or cross-hybridize to non-specific gene targets, core probes must be chosen to accurately represent changes in gene expression. Using Probe Select in the Affy package of Bioconductor, we implemented the Li-Wong model for probe selection (27) The algorithm developed by Li and Wong seeks to identify the largest subset of highly correlated probes to represent a single gene. They reason that the majority of probes targeting constitutive exons of a particular gene will have a comparable intensity profile across various different tissues, and reflect the overall mRNA abundance of that gene. Therefore, we downloaded the mouse exon array tissue panel dataset from Affymetrix consisting of 11 tissues (breast, cerebellum, heart, kidney, liver, muscle, pancreas, prostate, spleen, testes, and thyroid) each with three replicates. For each gene we calculated the Pearson correlation coefficient of the signal intensities of all possible pairs of probes across the 11 tissues (a total of 33 samples) and used that to perform average-linkage hierarchical clustering, which is visualized on a heatmap using R (Figure 18). Hierarchical clustering of our retina samples across the entire tissue panel indicates that our retina samples correlates most closely with retina tissue samples. When the retina samples alone were analyzed, $\mathrm{Tg}+$ samples clustered together and $\mathrm{Tg}$ - samples clustered for high and medium expressors (1178 and 1054). However, some of the positive samples from the low expressor line (1065) clustered with the Tg- samples. Since the $1065 \mathrm{Tg}+$ samples did not significantly differ from the Tg- samples, they were excluded from further analysis. This may be due to the fact that expression level of this line is so low that retina samples do not resemble those of the higher expressing lines until around P21. It is possible that samples from the low expressor 1065 line would cluster better if they were taken later.

Figure 16. Microarray Analysis

\section{A. Basic gene representation \\ Exon Array probes target each exonic region of a single gene}
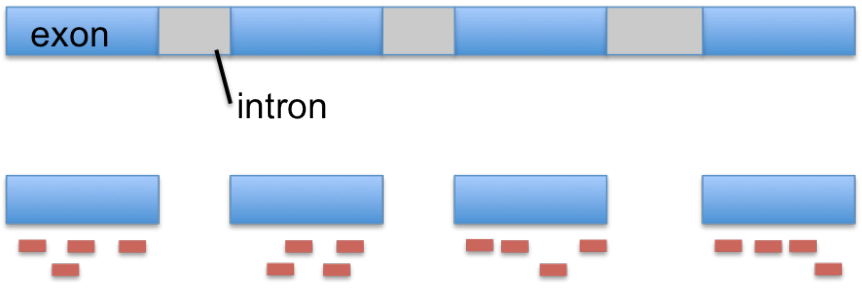

B. Experimental Design of Microarray showing 18 GeneChips. Retina samples are taken from 3 transgenic positive $(\mathrm{Tg}(+))$ mice and 3 transgenic negative $(\mathrm{Tg}$ $(-))$ mice for each line.

\begin{tabular}{cc|cc|cc}
\hline \multicolumn{2}{c|}{ High expressor } & \multicolumn{2}{|c|}{ Medium expressor } & \multicolumn{2}{c}{ Low expressor } \\
\hline $\mathrm{Tg}(-)$ & $\mathrm{Tg}(+)$ & $\mathrm{Tg}(-)$ & $\mathrm{Tg}(+)$ & $\mathrm{Tg}(-)$ & $\mathrm{Tg}(+)$ \\
I & I & I & I & I & I \\
II & II & II & II & II & II \\
III & III & III & III & III & III \\
\hline
\end{tabular}


Figure 17. Boxplot showing normalization across 51 sample sets, including the 18 retina samples and the Affymetrix array tissue panel dataset consisting of 11 tissues in triplicate.

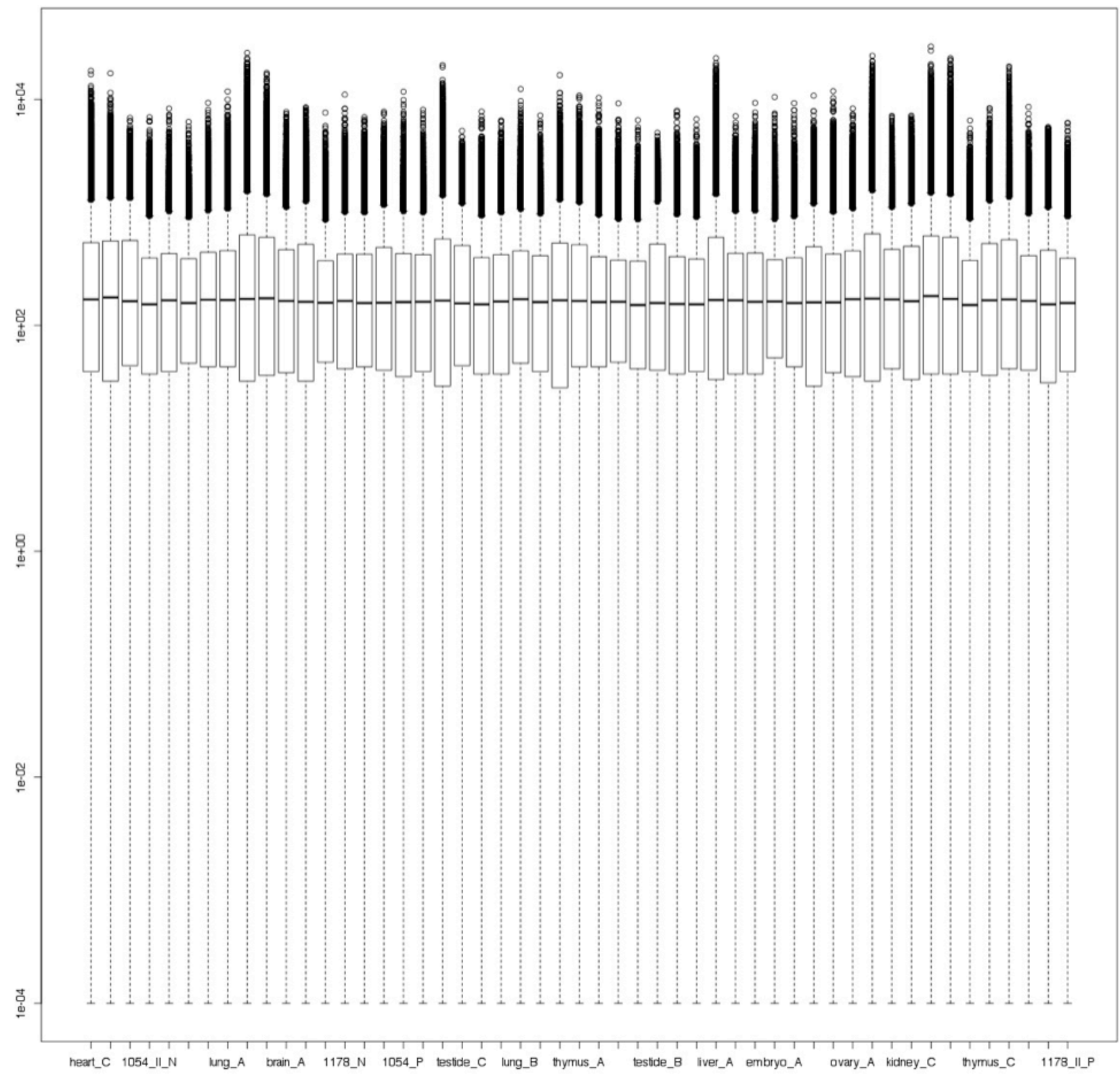


Figure 18. Probe correlation across Affymetrix Mouse Exon Array Tissue Panel
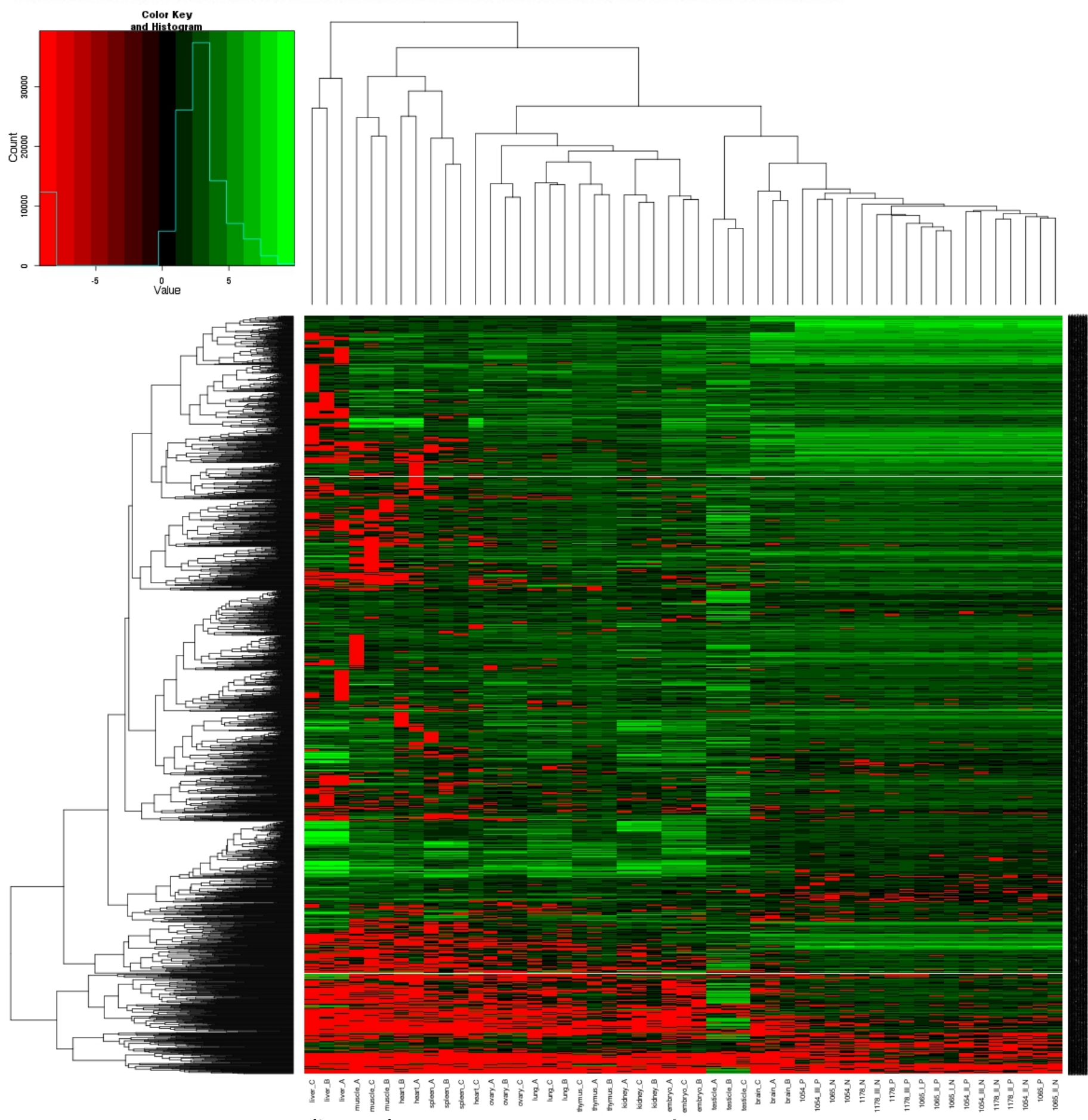

liver heart ovary lung thymus embryo brain $\operatorname{Tg}(-)$ and $\operatorname{Tg}(+)$ retina samples

Figure 18. Probe correlation across Affymetrix Mouse Exon Array Tissue Panel. Retina samples from three positive and three negative mice from each of the transgenic PhLPs mouse lines at post-natal day 10 were compared to the mouse exon array tissue panel dataset from Affymetrix consisting of 11 tissues (breast, cerebellum, heart, kidney, liver, muscle, pancreas, prostate, spleen, testes, and thyroid) each with three replicates. Hierarchical clustering was used to group together arrays that show correlated gene expression. 
Figure 19. Probe correlation of $\mathrm{Tg}+$ and $\mathrm{Tg}$ - mice from 1178 "high expressors", 1054 "medium expressors", and 1065 "low expressors" of PhLPs
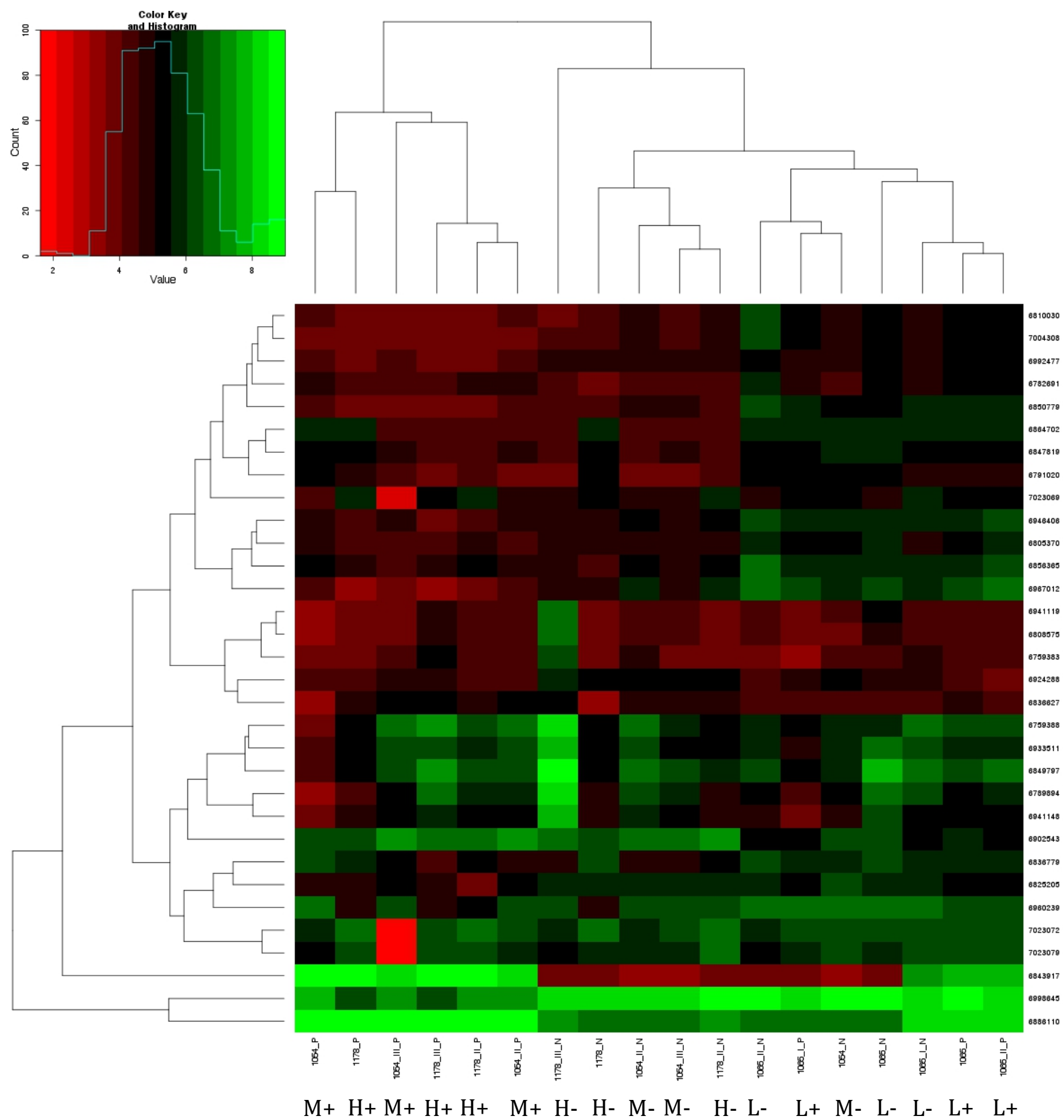

Figure 19. Gene expression was compared across transgenic positive and negative retinal samples from high (H), medium (M), and low (L) PhLPs expressor lines shown in figure 18. Hierarchical clustering was used to group together samples that show correlated gene expression. 
Figure 20. Genes with greater than 2 fold change in expression level

A. Downregulated genes

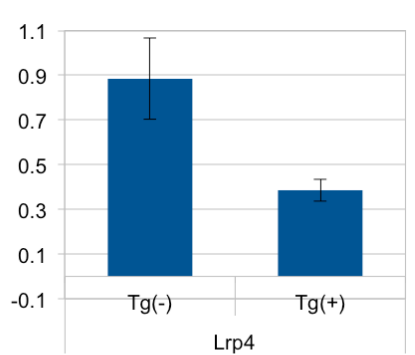

B. Upregulated genes

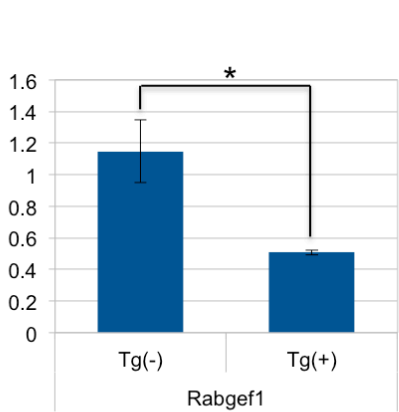

Microarray results were validated by measuring the change in expression of selected genes by quantitative PCR. Transgenic positive $\left(\mathrm{Tg}^{+}\right)$and negative ( $\left.\mathrm{Tg}-\right)$ retina samples were collected from the medium expressor PhLPs mice at post-natal day 10. Expression level for reach respective gene are normalized to the Tg- littermate.

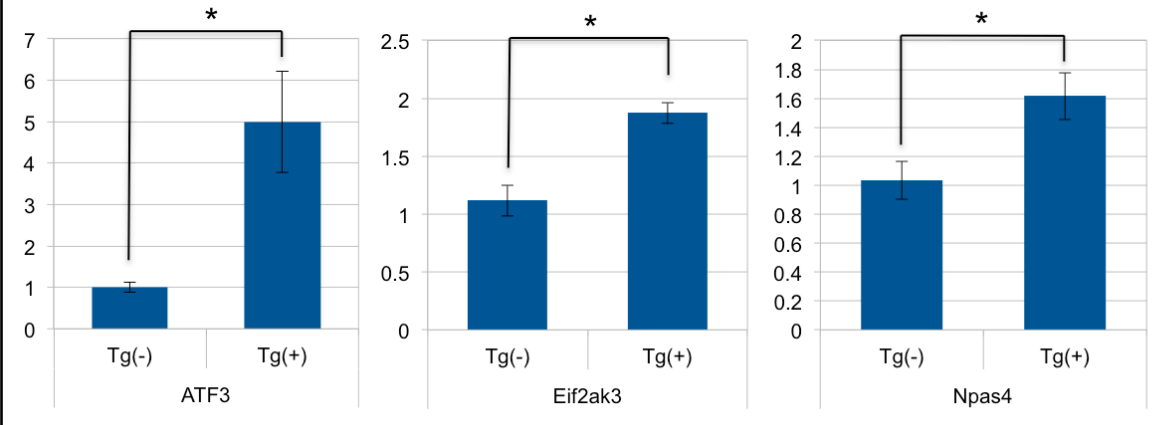

Genes expression from $\mathrm{Tg}(+)$ animals was compared to $\mathrm{Tg}(-)$ controls to identify genes that were affected by PhLPs. The change in gene expression was considered significant if the p-value was less than 0.05 and the change in expression was either greater than 1.5 or less than 0.5 . For both the high (1178) and medium (1054) expressor lines, only 160 genes had a significantly different expression level in $\mathrm{Tg}+$ samples. When all of the $\mathrm{Tg}+$ samples were compared to all of the Tg-samples, only 8 genes had a greater than 1.5 fold increase, and 9 genes were decreased by 0.5 or more (Table 1 ).

\section{Microarray Validation}

The results of the microarray were validated by quantitative Real Time PCR (qPCR). The microarray results show that $\mathrm{Gt}_{\mathrm{t}} \alpha_{1}$ was one of the genes that had been significantly decreased. In the combined analysis, it was reduced 0.2 fold, which is in agreement with previous qPCR results (Figure 15). Primers for 6 genes were designed for qPCR validation based on the microarray results. Lrp4 (low density lipoprotein receptor-related protein 4), and Rabgef1 (RAB guanine nucleotide exchange factor (GEF) 1) were shown to be downregulated by the microarray and were chosen for validation. Lrp4 is a potential cell surface endocytic receptor, which binds and internalizes extracellular ligands for degradation by lysosomes. Rabgef1 stimulates nucleotide exchange on RAB5A and is involved in endocytic membrane fusion and membrane trafficking of recycling endosomes. Additionally, 3 genes, including Atf 3 and two other genes that were shown to be upregulated based on microarray results were chosen, for the validation. Atf3 (activating transcription factor 3) binds the cAMP response element (CRE) sequence present in many viral and cellular promoters, and represses transcription from promoters with ATF sites. It is thought to repress transcription by stabilizing the binding of inhibitory cofactors at the promoter.

Npas4 (neuronal PAS domain protein 4) is primarily expressed in the limbic system and olfactory bulb where it acts as a transcriptional activator to activate expression of the drebrin gene. Npas4 had a 2.4 fold increase in the high expressor line (1178), but was not found to be significant ( $p$-value $=0.08$ ) due to one low value in the medium expressor line $(1054)$. Eif2ak3 (eukaryotic translation initiation factor 2 alpha kinase 3) was chosen because although the change in expression was found to be non-significant in the microarray ( $p$-value = 0.0583), it had a 2.1 fold change and is involved in the unfolded protein response (UPR). It will be interesting to determine if some of the observed photoreceptor defects are due to UPR from inhibition of CCT.

The results from the qPCR validation are in general agreement with the results of the microarray (Figure 20) because all of the genes show the same direction of expression change. However, there were some slight differences. The change in Lrp4 gene expression was not found to be significant ( $p$-value $=0.056$ ) due to variation in one $\mathrm{Tg}+$ sample. It is expected that with a larger sample size $(n>3)$ the difference in expression level will be significant. Additionally, the 
increase in gene expression was found to be significant for Eif2ak3 ( $p$-value $=0.009)$ and slightly significant for Npas4 $(p$ value $=0.048$ ) in the $\mathrm{qPCR}$ validation despite have a non-significant difference shown in the microarray. Overall, these results indicate that genes from $\mathrm{Tg}+$ samples showing greater than 1.5 or less than 2 fold change on the microarray are significantly different from Tg- samples, and the UPR may contribute to some of the photoreceptor degeneration.

Table 1

\begin{tabular}{|c|c|c|c|}
\hline$P$-value & Fold Change & Gene Name & Description \\
\hline 0.046 & 0.369 & Sgcg & sarcoglycan, gamma (dystrophin-associated glycoprotein) \\
\hline 0.026 & 0.380 & Lrp4 & low density lipoprotein receptor-related protein 4 \\
\hline 0.022 & 0.421 & Rabgef1 & RAB guanine nucleotide exchange factor (GEF) 1 \\
\hline 0.039 & 0.427 & Gm9909 & predicted gene 9909 \\
\hline 0.039 & 0.460 & Slc24a1 & solute carrier family 24 (sodium/potassium/calcium exchanger), member 1 \\
\hline 0.039 & 0.466 & Pfkfb2 & 6-phosphofructo-2-kinase/fructose-2,6-biphosphatase 2 \\
\hline 0.035 & 0.490 & Gucy2f & guanylate cyclase $2 f$ \\
\hline 0.022 & 0.500 & Sntb2 & syntrophin, basic 2 \\
\hline 0.048 & 0.519 & Rcvrn & recoverin \\
\hline 0.022 & 0.526 & Olfr1188 & olfactory receptor 1188 \\
\hline 0.040 & 0.530 & Trib1 & tribbles homolog 1 (Drosophila) \\
\hline 0.046 & 0.534 & Pla2r1 & phospholipase $\mathrm{A} 2$ receptor 1 \\
\hline 0.033 & 0.535 & 3632451006Rik & RIKEN cDNA 3632451006 gene \\
\hline 0.018 & 0.541 & Dhdh & dihydrodiol dehydrogenase (dimeric) \\
\hline 0.022 & 0.549 & Tmem108 & transmembrane protein 108 \\
\hline 0.040 & 0.555 & D730039F16Rik & RIKEN cDNA D730039F16 gene \\
\hline 0.045 & 0.601 & Gldc & glycine decarboxylase \\
\hline 0.041 & 0.604 & Plxna2 & plexin A2 \\
\hline 0.050 & 0.607 & Impg1 & interphotoreceptor matrix proteoglycan 1 \\
\hline 0.045 & 0.608 & $\mathrm{Nxnl2}$ & nucleoredoxin-like 2 \\
\hline 0.039 & 0.609 & Polg2 & polymerase (DNA directed), gamma 2, accessory subunit \\
\hline 0.045 & 0.609 & Plcd3 & phospholipase C, delta 3 \\
\hline 0.039 & 0.609 & Sema3f & sema domain, immunoglobulin domain (Ig), short basic domain, secreted, (semaphorin) 3F \\
\hline 0.039 & 0.610 & Ric8b & resistance to inhibitors of cholinesterase 8 homolog B (C. elegans) \\
\hline 0.043 & 0.614 & Jam2 & junction adhesion molecule 2 \\
\hline 0.039 & 0.614 & Gngt1 & guanine nucleotide binding protein (G protein), gamma transducing activity polypeptide 1 \\
\hline 0.049 & 0.627 & Wdr27 & WD repeat domain 27 \\
\hline 0.040 & 0.628 & Spata1 & spermatogenesis associated 1 \\
\hline 0.045 & 0.635 & Plekhg3 & pleckstrin homology domain containing, family G (with RhoGef domain) member 3 \\
\hline
\end{tabular}




\begin{tabular}{|c|c|c|c|}
\hline 0.039 & 0.642 & Ush2a & Usher syndrome 2A (autosomal recessive, mild) homolog (human) \\
\hline 0.043 & 0.643 & Tcfeb & transcription factor EB \\
\hline 0.022 & 0.644 & Arg2 & arginase type II \\
\hline 0.044 & 0.652 & Fam149a & family with sequence similarity 149, member $A$ \\
\hline 0.045 & 0.652 & Ndrg3 & $\mathrm{N}$-myc downstream regulated gene 3 \\
\hline 0.045 & 0.655 & Heg1 & HEG homolog 1 (zebrafish) \\
\hline 0.049 & 0.659 & Herc3 & hect domain and RLD 3 \\
\hline 0.045 & 0.670 & 5330431K02Rik & RIKEN cDNA 5330431K02 gene \\
\hline 0.049 & 0.674 & $\mathrm{Cc} 2 \mathrm{~d} 2 \mathrm{a}$ & coiled-coil and $\mathrm{C} 2$ domain containing $2 \mathrm{~A}$ \\
\hline 0.043 & 0.685 & Sgtb & small glutamine-rich tetratricopeptide repeat (TPR)-containing, beta \\
\hline 0.040 & 0.686 & Osbpl8 & oxysterol binding protein-like 8 \\
\hline 0.046 & 0.693 & Ap2a2 & adaptor protein complex AP-2, alpha 2 subunit \\
\hline 0.049 & 0.705 & Nme5 & non-metastatic cells 5 , protein expressed in (nucleoside-diphosphate kinase) \\
\hline 0.039 & 0.707 & Apex2 & apurinic/apyrimidinic endonuclease 2 \\
\hline 0.039 & 0.713 & Fbxo36 & F-box protein 36 \\
\hline 0.039 & 0.715 & Ikzf2 & IKAROS family zinc finger 2 \\
\hline 0.040 & 0.717 & Mak & male germ cell-associated kinase \\
\hline 0.017 & 0.722 & Sh3bgrl2 & SH3 domain binding glutamic acid-rich protein like 2 \\
\hline 0.039 & 0.723 & Ceacam12 & carcinoembryonic antigen-related cell adhesion molecule 12 \\
\hline 0.039 & 0.723 & Epb4.115 & erythrocyte protein band 4.1-like 5 \\
\hline 0.040 & 0.730 & Styx & serine/threonine/tyrosine interaction protein \\
\hline 0.039 & 0.742 & Glo1 & glyoxalase 1 \\
\hline 0.049 & 0.743 & Dmd & dystrophin, muscular dystrophy \\
\hline 0.039 & 0.744 & Hook1 & hook homolog 1 (Drosophila) \\
\hline 0.046 & 0.746 & Cdk6 & cyclin-dependent kinase 6 \\
\hline 0.041 & 0.749 & Mtap2 & microtubule-associated protein 2 \\
\hline 0.049 & 0.751 & Cacna1f & calcium channel, voltage-dependent, alpha 1F subunit \\
\hline 0.017 & 0.753 & Ppp2r5c & protein phosphatase 2 , regulatory subunit B (B56), gamma isoform \\
\hline 0.039 & 0.757 & 6530402F18Rik & RIKEN cDNA 6530402 F18 gene \\
\hline 0.033 & 0.758 & Capn7 & calpain 7 \\
\hline 0.022 & 0.763 & Crlf3 & cytokine receptor-like factor 3 \\
\hline 0.045 & 0.768 & Top1mt & DNA topoisomerase 1 , mitochondrial \\
\hline 0.043 & 0.768 & Ect2 & ect2 oncogene \\
\hline 0.039 & 0.774 & Kctd10 & potassium channel tetramerisation domain containing 10 \\
\hline 0.040 & 0.775 & Atp11b & ATPase, class VI, type $11 \mathrm{~B}$ \\
\hline 0.026 & 0.775 & Zfp322a & zinc finger protein $322 \mathrm{~A}$ \\
\hline 0.039 & 0.777 & Gramd1b & GRAM domain containing $1 \mathrm{~B}$ \\
\hline 0.040 & 0.787 & Rreb1 & ras responsive element binding protein 1 \\
\hline 0.046 & 0.790 & Akr1e1 & aldo-keto reductase family 1 , member E1 \\
\hline 0.039 & 0.791 & Trak2 & trafficking protein, kinesin binding 2 \\
\hline 0.045 & 0.792 & Pola1 & polymerase (DNA directed), alpha 1 \\
\hline 0.022 & 0.794 & Vps37a & vacuolar protein sorting 37A (yeast) \\
\hline 0.039 & 0.796 & Cspp1 & centrosome and spindle pole associated protein 1 \\
\hline 0.049 & 0.797 & Ypel2 & yippee-like 2 (Drosophila) \\
\hline 0.046 & 0.799 & Erlec1 & endoplasmic reticulum lectin 1 \\
\hline 0.045 & 0.799 & Prtg & protogenin homolog (Gallus gallus) \\
\hline 0.026 & 0.804 & Zfp281 & zinc finger protein 281 \\
\hline
\end{tabular}




\begin{tabular}{|c|c|c|c|}
\hline 0.039 & 0.804 & Usp12 & ubiquitin specific peptidase 12 \\
\hline 0.039 & 0.812 & Ofd1 & oral-facial-digital syndrome 1 gene homolog (human) \\
\hline 0.022 & 0.816 & Sh3glb1 & SH3-domain GRB2-like B1 (endophilin) \\
\hline 0.043 & 0.818 & Adamts16 & disintegrin-like and metallopeptidase (reprolysin type) with thrombospondin type 1 motif 16 \\
\hline 0.039 & 0.826 & Msh3 & mutS homolog 3 (E. coli) \\
\hline 0.043 & 0.831 & Zmym5 & zinc finger, MYM-type 5 \\
\hline 0.039 & 0.839 & Rassf8 & Ras association (RalGDS/AF-6) domain family ( $\mathrm{N}$-terminal) member 8 \\
\hline 0.039 & 0.840 & Gm3435 & predicted gene 3435 \\
\hline 0.041 & 0.841 & Rpl26 & ribosomal protein L26 \\
\hline 0.046 & 0.843 & Cplx2 & complexin 2 \\
\hline 0.040 & 0.854 & Ep300 & E1A binding protein $\mathrm{p} 300$ \\
\hline 0.040 & 0.857 & Pfdn5 & prefoldin 5 \\
\hline 0.041 & 0.862 & Zeb1 & zinc finger E-box binding homeobox 1 \\
\hline 0.046 & 0.864 & Zfp207 & zinc finger protein 207 \\
\hline 0.039 & 0.867 & Itsn2 & intersectin 2 \\
\hline 0.045 & 0.867 & Mtif2 & mitochondrial translational initiation factor 2 \\
\hline 0.049 & 0.874 & Zfp106 & zinc finger protein 106 \\
\hline 0.045 & 0.877 & Ank2 & ankyrin 2 , brain \\
\hline 0.041 & 0.878 & Csnk1g1 & casein kinase 1 , gamma 1 \\
\hline 0.043 & 0.878 & Kit & kit oncogene \\
\hline 0.045 & 0.882 & Rif1 & Rap1 interacting factor 1 homolog (yeast) \\
\hline 0.046 & 0.886 & Fmr1 & fragile $X$ mental retardation syndrome 1 homolog \\
\hline 0.049 & 0.895 & 2310008H09Rik & RIKEN cDNA $2310008 \mathrm{H} 09$ gene \\
\hline 0.039 & 0.907 & Ncor1 & nuclear receptor co-repressor 1 \\
\hline 0.045 & 0.908 & Rbm27 & RNA binding motif protein 27 \\
\hline 0.049 & 0.910 & Med14 & mediator complex subunit 14 \\
\hline 0.039 & 0.911 & Nfat5 & nuclear factor of activated T-cells 5 \\
\hline 0.041 & 0.912 & $\mathrm{Rb} 1 \mathrm{cc} 1$ & RB1-inducible coiled-coil 1 \\
\hline 0.039 & 0.917 & Naa16 & $\mathrm{N}$ (alpha)-acetyltransferase 16, NatA auxiliary subunit \\
\hline 0.040 & 0.935 & Gpatch3 & G patch domain containing 3 \\
\hline 0.048 & 1.062 & Robo1 & roundabout homolog 1 (Drosophila) \\
\hline 0.049 & 1.082 & E130112L23Rik & RIKEN cDNA E130112L23 gene \\
\hline 0.046 & 1.097 & Fras1 & Fraser syndrome 1 homolog (human) \\
\hline 0.046 & 1.119 & Zfp608 & zinc finger protein 608 \\
\hline 0.046 & 1.120 & Lpcat3 & lysophosphatidylcholine acyltransferase 3 \\
\hline 0.050 & 1.121 & Rhpn2 & rhophilin, Rho GTPase binding protein 2 \\
\hline 0.026 & 1.123 & Phldb1 & pleckstrin homology-like domain, family B, member 1 \\
\hline 0.040 & 1.133 & Diap1 & diaphanous homolog 1 (Drosophila) \\
\hline 0.043 & 1.143 & Rad50 & RAD50 homolog (S. cerevisiae) \\
\hline 0.049 & 1.152 & Nkain2 & $\mathrm{Na}+/ \mathrm{K}+$ transporting ATPase interacting 2 \\
\hline 0.039 & 1.160 & Ahcyl2 & S-adenosylhomocysteine hydrolase-like 2 \\
\hline 0.022 & 1.162 & Cyp1b1 & cytochrome P450, family 1 , subfamily b, polypeptide 1 \\
\hline 0.049 & 1.175 & Slc5a11 & solute carrier family 5 (sodium/glucose cotransporter), member 11 \\
\hline 0.043 & 1.210 & Prkcq & protein kinase $\mathrm{C}$, theta \\
\hline 0.043 & 1.217 & Rasa2 & RAS p21 protein activator 2 \\
\hline 0.040 & 1.218 & Plcd4 & phospholipase C, delta 4 \\
\hline 0.045 & 1.232 & D4Bwg0951e & DNA segment, Chr 4, Brigham \& Women's Genetics 0951 expressed \\
\hline
\end{tabular}




\begin{tabular}{|c|c|c|c|}
\hline 0.043 & 1.234 & Prmt8 & protein arginine $\mathrm{N}$-methyltransferase 8 \\
\hline 0.045 & 1.237 & Dnahc11 & dynein, axonemal, heavy chain 11 \\
\hline 0.045 & 1.294 & Jph1 & junctophilin 1 \\
\hline 0.022 & 1.294 & S1pr3 & sphingosine-1-phosphate receptor 3 \\
\hline 0.049 & 1.295 & Slc7a2 & solute carrier family 7 (cationic amino acid transporter, $y+$ system), member 2 \\
\hline 0.035 & 1.296 & Lsm10 & U7 snRNP-specific Sm-like protein LSM10 \\
\hline 0.046 & 1.324 & Insl5 & insulin-like 5 \\
\hline 0.027 & 1.333 & Gdpd2 & glycerophosphodiester phosphodiesterase domain containing 2 \\
\hline 0.049 & 1.352 & Dnaic1 & dynein, axonemal, intermediate chain 1 \\
\hline 0.039 & 1.355 & Mrc2 & mannose receptor, $C$ type 2 \\
\hline 0.043 & 1.373 & Ticam1 & toll-like receptor adaptor molecule 1 \\
\hline 0.024 & 1.382 & Insl5 & insulin-like 5 \\
\hline 0.043 & 1.385 & Tmod1 & tropomodulin 1 \\
\hline 0.022 & 1.386 & Zmynd10 & zinc finger, MYND domain containing 10 \\
\hline 0.022 & 1.392 & Pycr1 & pyrroline-5-carboxylate reductase 1 \\
\hline 0.039 & 1.442 & $\mathrm{H} 19$ & H19 fetal liver mRNA \\
\hline 0.045 & 1.456 & Accn5 & amiloride-sensitive cation channel 5 , intestinal \\
\hline 0.045 & 1.490 & Apol7b & apolipoprotein L 7b \\
\hline 0.001 & 1.603 & Abca1 & ATP-binding cassette, sub-family A ( $A B C 1$ ), member 1 \\
\hline 0.039 & 1.648 & Efcab8 & EF-hand calcium binding domain 8 \\
\hline 0.039 & 1.727 & Klf10 & Kruppel-like factor 10 \\
\hline 0.039 & 1.889 & Wdr16 & WD repeat domain 16 \\
\hline 0.035 & 1.960 & Pmp2 & peripheral myelin protein 2 \\
\hline 0.043 & 3.747 & Atf3 & activating transcription factor 3 \\
\hline 0.022 & 5.954 & Pdcl & phosducin-like \\
\hline
\end{tabular}

\section{Gene Ontology Analysis}

Gene Ontology (GO) analysis was performed to see what pathways or cellular functions were enriched based on the microarray results. Using the web-based on-line tool WebGestalt (WEB-based GEne SeT AnaLysis Toolkit), the genes that changed significantly in Tg+ animals were compared back to the annotated Affymetrix Mouse Exon Array 1.0 for enrichment analysis. The results from the WebGestalt enrichment analysis were organized into a "GO Tree", which is based on the GO DAG (Directed Acyclic Graph) in order to visualize enrichment categories, enriched gene numbers, and the statistical significance level (Figure 21). An enriched DAG shows GO categories with enriched gene numbers (in red) and their non-enriched parents (in black). Based on the microarray experiment, a total of 160 genes had significant expression changes in Tg+ samples from both the high (1178) and medium (1054) expressor lines. Of these genes, 55 had expression changes by 1.5 fold or more. These genes were organized into a GO Tree under 3 broad categories for biological process, molecular function, and cellular process.

The gene categories that were significantly enriched $(\mathrm{p}<0.05)$ upon PhLPs expression fell under the 'biological process' subheading. A few categories that were similar were also made up of the same genes so All of the categories that most of the enriched GO categories identified for this gene set could be placed into one of five categories related to response to stimulus, phototransduction, eye and sensory organ development, membrane organization \& endocytosis, and sensory/visual perception. Under the category 'response to stimulus' the genes Gngt1, Gnat1, and Rcvrn were also found in 'response to radiation', 'detection of abiotic stimulus', 'detection of external stimulus', 'detection of stimulus', 'response to light stimulus', 'response to abiotic stimulus', and 'phototransduction'. Similarly, the same three genes, Rpgrip, Gngt1, and Gnat1, make up the categories 'eye photoreceptor development', 'photoreceptor cell differentiation', 'photoreceptor cell development', 'eye development', 'neuron development'. In addition, the genes Lrp4, Abca1, Pla2r1, and Rabgef1 make up the 'endocytosis' category as well as 'membrane invagination', 'vesicle-mediated transport', and 'membrane organization'. Lastly, the 'sensory perception of light' and 'visual perception' categories were made up of the genes Pdcl, Rpgrip1, Rcvrn, Ush2a, Gucy2f, and Gnat1. Although 'spermatid development' was listed as a significantly 
enriched category, it is made up of only two genes, Dhh and Prm1. However, the significant upregulation in gene expression of Prm1 (protamine 1) that was shown in the microarray analysis may just be an artifact because the polyadenylation sequence of the transgene was derived from Prm1. Further validation tests using qPCR have indicated that the levels of Prm1 do not change significantly.

What is noticeably absent from the enrichment analysis is an involvement of genes in apoptotic or the UPR. Since the minimum number of genes required to show enrichment in a single category was only set to 2, it is unlikely that these pathways play a role in CCT's connection to G-protein expression. A few individual genes that appeared in many categories of the GO Tree analysis were Lrp4, Rabgef1, Rcvrn, Ush2a, and Rpgrip1. Lrp4 (low density lipoprotein receptor-related protein 4) and Rabgef1 (RAB guanine nucleotide exchange factor (GEF) 1) are both involved in membrane organization and invagination, and Lrp4 is also involved in cellular development. The Rcvrn (recoverin) gene encodes a member of the recoverin family of neuronal calcium sensors and is thought to prolong the termination of the phototransduction cascade in the retina by blocking the phosphorylation of photo-activated rhodopsin. Two very interesting genes are Ush2a (Usher syndrome 2A homolog) and Rpgrip1 (retinitis pigmentosa GTPase regulator interacting protein 1), which are both involved in photoreceptor development, visual perception, and cell projection. Usher syndrome is a genetic disorder that is characterized by deafness and progressive loss of vision. The three different types of Usher syndrome are thought to be responsible for 3 percent to 6 percent of all childhood deafness and about 50 percent of deaf-blindness in adults. Interestingly, the loss of vision is due to the eye disease, retinitis pigmentosa (RP), which causes photoreceptor degeneration. Although Ush2a expression was only increased by 0.64 fold (Table 1), it is tempting to make a connection between CCT inhibition and gene expression changes that are seen in a progressive eye disease. Overall, these results support the role of CCT in G-protein expression and signaling because when CCT is inhibited by PhLPs, the genes involved in visual processing and photoreceptor development are significantly impacted. 
Figure 21. Gene Ontologv functional enrichment analvsis

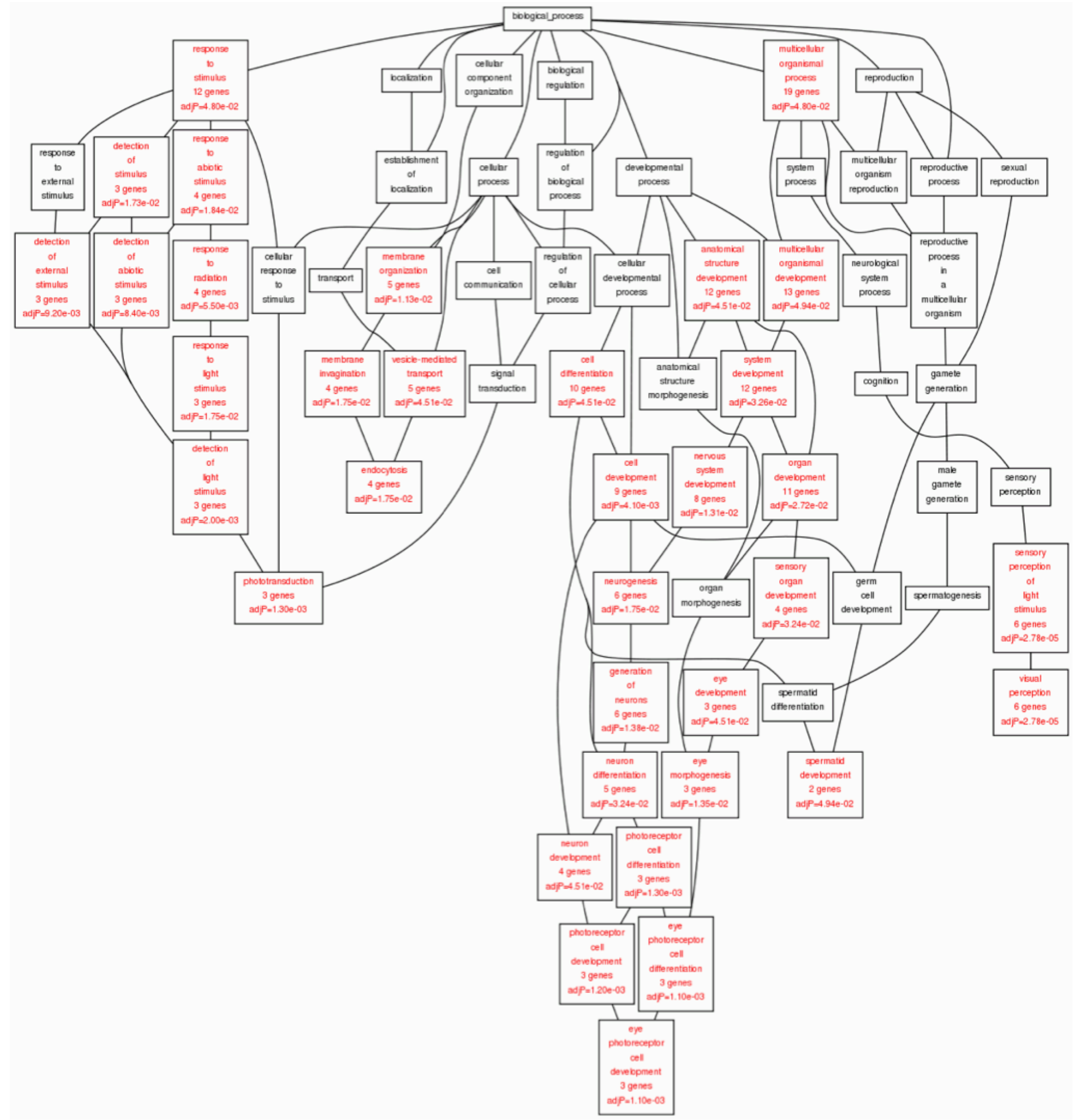


Inducible expression of PhLPs (iPhLPs)

In young mice, the expression of rhodopsin occurs around age P5 and triggers the development of rod photoreceptor outer segments. Inhibition of CCT during this critical period could affect specific molecular or signaling events related to development. A major limitation of the high (1178), medium (1054), and low (1065) expressor lines is the inability to control PhLPs expression. Expression of PhLPs in these lines began at P10 with the expression of rhodopsin. Because we could only identify the transcriptional CCT feedback in the underdeveloped photoreceptors of neonatal mice constitutively expression PhLPs we couldn't determine whether the inhibition of CCT might cause development changes. To overcome this limitation, transgenic models with the regulated tetracycline-dependent PhLPs expression have been developed in our laboratory in order to study the effect of CCT suppression on the Gt $\alpha_{1}$ gene (Gnat1) activity in retinas of adult mice.

We have developed six lines of transgenic mice that have tetracyclin-inducible PhLPs expression (iPhLPs) to allow us to study the effect of CCT suppression on Gnat1 activity in retinas of adult mice. These mice were generated by crossing transgenic TRE-Tight- $41-83$ PhLP-FLAG mice (expressing PhLPs), with mice containing a reverse tetracyclinedependent transactivator (rtTA) under the control of an opsin promoter. The result of this cross is a mouse line carrying both the TRE-Tight- $41-83$ PhLP-FLAG as well as the rtTA transgene (Figure 22A). After age P5 rtTA is continuously produced in retinal photoreceptor cells where the opsin promoter is active. In order for the TRE-Tight promoter to become active, it must be bound by both rtTA and tetracycline. Therefore, in this inducible model administration of the tetracycline analog, doxycycline, drives PhLPs expression specifically in photoreceptor cells.

Several lines of iPhLPs were generated and tested for PhLPs expression by FLAG immunoprecipitation (IP) and western blotting for $\alpha$-FLAG. Out of these lines, two lines (line 2339 and 2347) were chosen for further experimentation because they had the highest level of PhLPs expression. Mice from these lines were genotyped to identify individuals that were positive for the iPhLPs transgene. These PhLPs positive mice were then given doxycycline to induce expression of PhLPs in retinal photoreceptors. Initially, doxycycline was administered through drinking water at either 1.0 or 10 $\mathrm{mg} / \mathrm{ml}$ for 10 days, but there was some variability in PhLPs induction. In order to reduce the variability, all mice were given $10 \mathrm{mg} / \mathrm{ml}$ doxycycline $+50 \mathrm{mg} / \mathrm{ml}$ sucrose in their drinking water for 1, 3, or 9 days (figure 22B upper panel). Because PhLPs expression remained highly variable and occurred with low frequency, $0.1 \mathrm{ml}$ doxycycline $(10 \mathrm{mg} / \mathrm{ml}) \mathrm{was}$ administered by intraperitoneal injection every $24 \mathrm{hrs}$ for two days (figure 22B, lower panel). Despite the efforts to standardize the amount of doxycycline that each animal received, the individual variability in PhLPs expression even among

Figure 22. Inducible expression of PhLPs in transgenic mice

A.

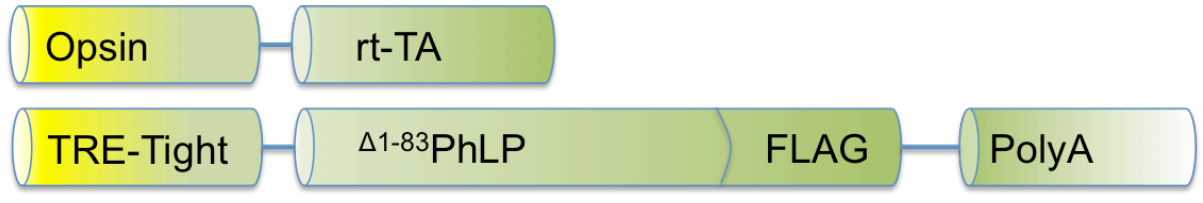

B. DOXY: drinking water days

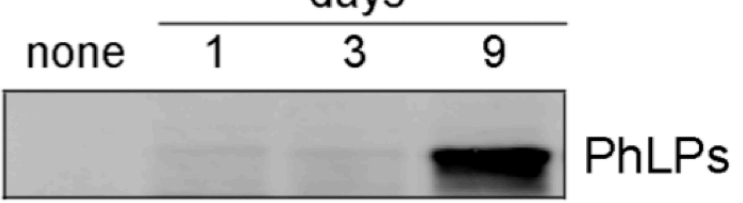

DOXY: IP injections

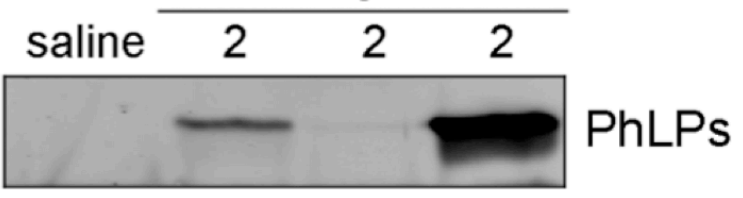

A. Transgene utilized for the inducible expression of FLAG-tagged PhLPs ( $\triangle 1-83 \mathrm{PhLP})$ in mouse photoreceptors. An opsin promoter drives expression of the reverse tetracycline transactivator (rt-TA) while a commercial tetracycline responsive promoter, TRE-Tight (Clontech), to a mouse PhLPs cDNA containing a C-terminal FLAG tag. B PhLPs induction was monitored by immunoprecipitation of retinal samples with anti-FLAG antibody. Upper panel, PhLPs expression was first induced by administering doxycycline in drinking water 5 $\mu \mathrm{g} / \mathrm{ml}$ doxycycline $+50 \mu \mathrm{g} / \mu \mathrm{l}$ sucrose for 10 days. Lower panel, in order to decrease variablility in PhLPs expression, $1 \mathrm{mg}$ doxycycline was administered through interperitoneal injection twice a day for two day. 
littermates remained high.

In order to test whether

PhLPs induced CCT inhibition

in adult mice has the same

effect on G-protein levels in

mice at P10, the levels of Gnat1,

Gnb1, and Gng1 were

measured in adult iPhLPs mice.

iPhLPs positive mice mice were identified by genotyping and given $0.1 \mathrm{ml}$ doxycycline (10 $\mathrm{mg} / \mathrm{ml}$ ) by intraperitoneal injection every $24 \mathrm{hrs}$ for five days. After the induction period, mice were sacrificed and one retina was used to identify mice that expressed PhLPs while the other eye from the same mouse was used to measure G-protein levels. Even after 5 days of doxycycline

injects not all of the animals that were identified as PhLPs positive by genotyping expressed a high enough amount of PhLPs to be detectable by IP. The mice that were PhLPs positive but did not express sufficient PhLPs, referred to as "nonexpressors", were used as a negative control (N/Exp in figure 23). The results shown in figure 23 compare the change in expression of $G_{t} \alpha_{1}, G \beta_{1}$, and $G \gamma_{1}$ normalized to HPRT from the two iPhLPs lines to non-expressors from $2347(n=3)$. $G \beta_{1}$ and $\mathrm{G} \gamma_{1}$ levels do not change significantly compared to control. However, $\mathrm{G}_{\mathrm{t}} \alpha_{1}$ had a 9 fold reduction in the 2339 line and a 6 fold reduction in the 2347 line. The results from this set of experiments is in general agreement with the results from the constitutive line, indicating that the reduction of G-protein levels by CCT inhibition is not dependent on a developmental process.

\section{Discussion}

The objective of this project has been to investigate the role of CCT in the biosynthesis and regulation of heterotrimeric $G$ proteins. I set out to build upon the initial data from our lab indicating that the inhibition of CCT affects $\mathrm{Gt}_{1} \alpha_{1}, G \beta_{1}$, and $G \gamma_{1}$ at the protein level as well as the mRNA transcript level. The inducible PhLPs (iPhLPs) mouse lines were generated in an attempt to find a connection between CCT and G-protein expression that is dependent on a developmental process. Despite the low frequency of PhLPs induction and the high variability among individual animals I was able to complete one set of experiments. Because the reduction in G-protein levels from these adult animals initially appears to be the same as those seen in younger mice from the constitutive lines, it appears that mature photoreceptors as just as susceptible as developing photoreceptors. However, due to the high variability in PhLPs expression, this experiment will need to be repeated.

PhLPs is a potent inhibitor of CCT, which is responsible for folding a wide variety of proteins with many diverse functions. Based on this, one might think that the most significant effect of PhLPs expression would be triggering of the unfolded protein response (UPR). However, the results of the microarray and Gene Ontology (GO) analysis have indicated that this is not the case. Very few genes are significantly affected by the inhibition of CCT by PhLPs based on the microarray results. Only 160 genes had changes in gene expression that were significant different in either the high (1178) or medium (1054) expressor lines of mice constitutively expressing PhLPs. Out of these 160 genes only 9 genes had expression levels that were decreased by more than 2 fold or increased more than 1.5 fold. Collectively these experiments indicate that CCT plays an essential role in the visual process. The genes that are most heavily impacted by its inhibition are involved in visual perception or cellular organization. Gnat1 stood out in the microarray results because it had the 
largest decrease in mRNA expression. Despite the fact that these results were strikingly specific, they still offered no direct connection between CCT and Ga.

In the future, it may be prudent to design experiments that connect the resulting changes in gene expression due to CCT inhibition with loss of photoreceptor cells. It will be interesting to see if inducing PhLPs in adult mice will lead to the same degree of photoreceptor death that was seen in the constitutive lines of younger animals. Because many of the same genes participate in numerous biological processes as indicated by the GO analysis, it is possible that the Ga loss due to PhLPs expression causes more significant cell death in developing photoreceptors than in adult photoreceptors. This could be tested by doing functional experiments such as an electroretinogram (ERG) slides and histology experiments using photoreceptors of inducible mice to support the previous findings. This may lead to discovering a connection between CCT and G $\alpha$. 


\section{EXPERIMENTAL PROCEDURES}

\section{Generation of PhLPs Transgenic Mice}

Three lines of transgenic mice were generated (28)to constitutively express PhLPs in rod photoreceptor cells. To prepare the dominant negative form of phosducin-like protein lacking the first 83 amino acids and containing C-terminal FLAG tag, total RNA was first isolated from the retina of a 129/SV mouse using the Absolutely RNA Miniprep Kit (Stratagene, La Jolla, CA), and the RNA was reverse transcribed using the mouse PhLP gene-specific RT primer 5'-ACT AAA TGA GAC TAC AA with the AccuScript High Fidelity 1st Strand cDNA Synthesis Kit (Stratagene; \#200820). A PCR, using the PfuUltra Hotstart PCR Master Mix (Stratagene; \#600630), was used to amplify the coding sequence of PhLP from the subsequent cDNA, beginning with the 84 th codon, thereby creating ${ }^{11-83} \mathrm{PhLP}$ (PhLPs). At the same time, a Sall site and a Kozak sequence (GCCACCATGG) were added to the 5' end, and a FLAG sequence, followed by a BamHI site and two new stop codons, were added to the $3^{\prime}$ end of PhLPs. Because the 84th codon of PhLP happens to be ATG, no additional start codon was needed. The following primers were used during PCR to achieve these changes: forward primer $5^{\prime}$-GAG AGT CGA CGC CAC CAT GGA GCG GCT GAT CAA AAA G and reverse primers 5'- CTT GTC ATC GTC GTC CTT GTA ATC ATC TAT TTC CAG ATC GCT GTC TTC and 5'- GCC TGG ATC CCT ACT ACT TGT CAT CGT CGT CCT TGT AAT C. Two separate PCR reactions were performed to keep the primers from getting too large. Both reactions were done using the same forward primer, whereas the first reaction was done with the first reverse primer shown and the second reaction with the second reverse primer shown. After each reaction, PCR products were run on an agarose gel, and the appropriate product band cut out and purified with the Wizard SV Gel and PCR Clean-Up System (Promega, Madison, WI; A9281). The final PCR product was cloned into the pBAM4.2 vector containing a $4.4 \mathrm{~kb}$ mouse rhodopsin promoter and a mouse protamine I polyadenylation sequence (29). Briefly, both the final PCR product and the pBAM4.2 vector were digested with SalI and BamHI restriction endonucleases, gel purified, and ligated so the Kozak-PhLPs-FLAG sequence would be inserted following the mouse rhodopsin promoter and before the mouse protamine I poly(A) sequence, creating pRhop4.4k-PhLPs. The integrity of the construct was confirmed by sequence analysis, and the final transgene (4.4 kb rhodopsin promoter-Kozak-PhLPs-FLAG-MPI poly(A)) was removed from pRhop4.4k-PhLPs by digestion with restriction endonucleases KpnI and SpeI, gel purified, and used for mouse pronuclear injections at the WVU Transgenic Animal Core Facility. Founder mice carrying PhLPs-FLAG were selected by PCR genotyping of tail genomic DNA using the following primers: forward primer 5'-GTG AAT TGA TTG GCA ATT TTG TTC G and reverse primer 5'- ACT ACT TGT CAT CGT CGT CC.

Three lines of mice constitutively producing PhLPs were generated and designated 1178, 1054, and 1065. Protein expression of PhLPs-FLAG in the retinas was confirmed and quantified by immunoblotting using anti-FLAG antibody. The 1178 line had highest expression and is referred to as the high expressor, 1065 is the low expressor, and 1054 is the middle expressor of PhLPs. Colonies were established and maintained by crossing PhLPs-FLAG mice with 129/SV mice (Charles River, Wilmington, MA). All experiments involving animals were performed according to the procedures approved by the Animal Care and Use Committees of West Virginia University and University of Minnesota.

\section{Generation of the inducible PhLPs-i transgenic mice}

In order to control the onset of PhLPs expression and CCT suppression, we have generated transgenic mice with the ability to induce expression of PhLPs (PhLPs-i) using a reverse tetracycline-dependent transactivator (rtTA). Transgenic TRE-Tight-PhLPs-FLAG mice were generated by adding a commercial tetracycline responsive promoter, TRETight (Clontech), to a mouse PhLPs cDNA containing a C-terminal FLAG tag. These transgenic TRE-Tight-PhLPs-FLAG were crossed with opsin-rtTA mice (Tg(opsin-rtTA) +/+), generously provided by Drs. Zack and Campochiaro (Johns Hopkins University). Using this model, six transgenic PhLPs-i lines have been generated at the WVU Transgenic Animal Core Facility. Because the reverse tetracycline-dependent transactivator (rtTA) is under the control of an opsin promoter, progeny of this cross express rtTA in photoreceptors. Following administration of the stable tetracycline analog, doxycycline, the TRE-Tight promoter will drive expression of PhLPs-FLAG in photoreceptors. Doxycycline was administered through drinking water $(5 \mu \mathrm{g} / \mathrm{ml}$ doxycycline $+50 \mu \mathrm{g} / \mu \mathrm{l}$ sucrose for 10 days $)$ and interperitoneal injection ( $0.1 \mathrm{ml}$ twice a day for 5 days), and was optimized by monitoring PhLPs protein levels through immunoprecipitation (IP) and western blot of $\alpha$-FLAG. In two of the lines, progeny positive for both transgenes (Tg(PhLPs-i)+/-; $\operatorname{Tg}($ opsin-rtTA) +/-) express amounts of PhLPs comparable to the 1054 (medium PhLPs expressor) constitutive line. Both models allow expression of PhLPs in mature photoreceptors. 
Immunoprecipitation and Western Blot

Single retinas were homogenized by short ultrasonic pulses in $0.2 \mathrm{ml}$ of RIPA buffer (Sigma; R0278). Cell membranes and insoluble parts were cleared by centrifugation, and supernatant was incubated with $10 \mu \mathrm{l}$ of anti-FLAG M2-Agarose (Sigma; A2220), for $1 \mathrm{~h}$ at room temperature. Beads were washed 3 times with $0.2 \mathrm{ml}$ of RIPA buffer. Proteins bound to the FLAG-Agarose beads were eluted using 1\% USB (20 mM Tris-HCl, pH 7.5, $8 \mathrm{M}$ urea, 4.5\% SDS), and loaded onto a 10\% Agarose gel. After the bands were separated, they were transferred to an Immobilon-FL PVDF membrane (Millipore) in Towbin buffer containing $25 \mathrm{mM}$ Tris, $192 \mathrm{mM}$ Glycine, and 10\% (v/v) methanol for $1.5 \mathrm{~h}$ at 0.25 A.

Western Blot - After transfer, the membrane was blocked with Odyssey non-mammalian protein blocking buffer (LI-COR; 927-40000) for 10 minutes at room temperature. The membrane was then probed with rabbit $\alpha$-FLAG (Rockland; 600-401-383) at 1:5,000 dilution in 1\% PBS (Phosphate Buffered Saline solution, Fisher; BP399-4) with 0.01\% Tween 20 (Sigma; P7949) for 1 hour at room temperature. Before the secondary antibody was applied, the membrane was washed three times with 1\% PBS with 0.01\% Tween 20 for five minutes. Goat-anti rabbit IgG Alexa Fluor 680 (Invitrogen; A21109) was then applied at 1:50,000 dilution in 1\% PBS with $0.01 \%$ Tween 20 for 45 minutes at room temperature. The membrane was washed three times with $1 \%$ PBS with $0.01 \%$ Tween 20 for five minutes before bands were visualized using an Odyssey Infrared Imaging System (LI-COR Biosciences, Lincoln, NE) according to the manufacturer's protocols.

\section{RNA Isolation}

Single retinas were collected from the constitutive PhLPs mouse lines at postnatal day 10 and 11 (P10 and P11), while samples from the inducible PhLPs (iPhLPs) mouse lines were taken from adult animals (8-10 weeks of age). Total RNA was isolated from each of these retinas using the Absolutely RNA Miniprep kit (Agilent Technologies), according to the manufacturer's protocol for small samples, and including two DNAse treatments. The final concentration of total RNA (A260) was quantified using a NanoDrop ND-1000 spectrophotometer (NanoDrop Technologies).

qRT-PCR

Using a two-step qRT-PCR process, cDNA from each retina was generated from 5ng of total RNA using the AffinityScript QPCR cDNA Synthesis kit (Agilent Technologies) and oligo(dT) primers. No template reactions served as negative controls. qRT-PCR experiments were performed in triplicate with $0.5 n g$ of cDNA using the Brilliant II SYBR ${ }^{\circledR}$ Green QPCR Master Mix (Agilent Technologies), reference dye, and $200 \mathrm{nM}$ of each primer. Reactions were incubated at $95^{\circ} \mathrm{C}$ for $10 \mathrm{~min}$, and then cycled $27 \mathrm{X}$ at $95^{\circ} \mathrm{C}$ for $30 \mathrm{~s}, 55^{\circ} \mathrm{C}$ for $60 \mathrm{~s}$, and $72^{\circ} \mathrm{C}$ for $30 \mathrm{~s}$ using a Stratagene Mx3000P ${ }^{\mathrm{TM}}$ realtime PCR system. A melting curve analysis was added at the end to verify a single product from each reaction, and the fluorescence was recorded during every QPCR cycle at both the annealing step $\left(55^{\circ} \mathrm{C}\right)$ and the extension step $\left(72^{\circ} \mathrm{C}\right)$. Fluorescence values were analyzed using MxPro QPCR Software version 4.10 and amplification thresholds were normalized to those of GAPDH.

Primers for mouse Gnat1, Gnb1, and Gngt1 (Table 1) were designed using GenBank mouse mRNA sequences and PrimerQuest software (http://scitools.idtdna.com/Primerquest/) and selected to amplify products crossing exon-exon boundaries. The primers were synthesized and HPLC-purified by Integrated DNA Technologies (IDT) (Coralville, IA). Primer were optimized for each reaction prior to running qRT-PCR experiments. The primer sequences used were as follows:

\begin{tabular}{|l|l|l|}
\hline & Forward 5' -3 $^{\prime}$ & Reverse $5^{\prime}-3^{\prime}$ \\
\hline Gnat1 & TGCCATCATCTACGGCAACACTCT & CTTGGGCATTGTGCCTTCCTCAAT \\
\hline Gnb1 & AGAATCCAAATGCGGACCAGGAGA & ACCACAGGCCACATAATTCCCAGA \\
\hline Gng1 & TGCCAGTGATCAACATCGAAGACC & TCACACAGCCTCCTTTGAGTTCCT \\
\hline GAPDH & GACTTCAACAGCAACTCCCAC & TCCACCACCCTGTTGCTGTA \\
\hline
\end{tabular}

\section{Microarray}

Affymetrix GeneChip Whole Transcript (WT) Sense Targeting Labeling Assay was used on Exon Array GeneChips. Whole retinas from PhLPs positive transgenic animals $(\mathrm{Tg}(+))$ were compared to PhLPs negative littermate controls ( $\operatorname{Tg}(-))$ for each of the three constitutive lines (1178 "high" expressor, 1054 "medium" expressor, and 1065 "low" expressor) in triplicate on individual chips for a total of 18 arrays. The microarray chips were analyzed using a GeneChip 
Scanner 3000 7G Plus (Affymetrix). The resulting files generated from the Affymetrix chips were analyzed in R using Bioconductor. Background subtraction and normalization were performed using GeneBASE (Genelevel Background Adjusted Selected probe Expression). Briefly, the fluorescence signals from antigenomic background probes were subtracted from core probes. The background-adjusted core probes were then normalized using a simple multiplicative scaling factor so that the median value equals 100.

Gene expression indexes for each gene were computed using the Li-Wong model $(27,30)$ via the affy package of Bioconductor (http://www.bioconductor.org/). The Li-Wong algorithm seeks to identify the core probes that best reflect the overall mRNA abundance by identifying the largest subset of highly correlated probes that correspond to exons across various mouse tissues. For this purpose, our samples were compared to the Mouse Exon 1.0 ST Array tissue panel dataset (http://www.affymetrix.com/support/technical/sample_data/exon_array_data.affx) consisting of 11 mouse tissues (breast, cerebellum, heart, kidney, liver, muscle, pancreas, prostate, spleen, testes, and thyroid) each with three replicates. For each transcript cluster, we calculated the Pearson correlation coefficient of the signal intensities of all possible pairs of probes across the 11 tissues (a total of 33 samples). We visualized the correlation matrix of the probe intensities as a heatmap using R (http:///www.r-project.org).

Differences in gene expression between $\operatorname{Tg}(+)$ and $\operatorname{Tg}(-)$ were calculated using a two-tailed student's t-test. Changes were considered significant if the p-value was less than 0.05 and the fold change increased or decreased by more than 2 fold. Gene Ontology (GO) analysis of the annotated gene list was performed using the web-based on-line tool WebGestalt (WEB-based GEne SeT AnaLysis Toolkit). A list of the Entrez Gene ID numbers of differentially expressed genes were uploaded to http://bioinfo.vanderbilt.edu/webgestalt/ and analyzed using the entire annotated dataset as a reference set for enrichment analysis. A hypergeometric statistical method was used with bonferroni adjustment. The significance level was set at 0.05 and the minimum number of genes per category was set at 2 .

Genes were validated using qPCR as stated above. Using the Stratagene MX3000P thermocycler, reactions were incubated at $95^{\circ} \mathrm{C}$ for $10 \mathrm{~min}$, and then cycled for 40 cycles at $95^{\circ} \mathrm{C}$ for $30 \mathrm{~s}$, and $64^{\circ} \mathrm{C}$ for $30 \mathrm{~s}$. Products were run on an agarose gel to check for specificity.

\begin{tabular}{|l|l|l|}
\hline & Forward 5 $^{\prime}$ - $^{\prime}$ & Reverse $^{\prime}{ }^{\prime}-3^{\prime}$ \\
\hline Sgcg & AAAGTGGGTGCCCAGATGGTAGAA & AAACCCACAGTTTCAGCATCCAGC \\
\hline Lrp4 & TGACAGCAGTGACGAAAGTCCACA & TTACATCCTGGCACGTTCTCCCAT \\
\hline Rabgef1 & ACCTTCCACAAGACAGGCCAAGAA & ACTTTCTCTGGAGGCACTTTCCCA \\
\hline Eif2ak3 & AGCCAGAGGTGTTTGGGAACAAGA & ACCGAAGTTCAAAGTGGCCAACAC \\
\hline Npas4 & TCAGGCTGAAATGGTGGTGAGACT & TGAAGTCCAGCTCTTTGGAGGGTT \\
\hline Atf3 & GTCAGTTACCGTCAACAACAGACC & GCAGGCACTCTGTCTTCTCC \\
\hline HPRT & CAGGCCAGACTTTGTTGGAT & GGACGCAGCAACTGACATT \\
\hline
\end{tabular}

\section{Molecular modeling}

3D structures were obtained from the Research Collaboratory for Structural Bioinformatics (RCSB) Protein Databank (PDB) http://www.rcsb.org. Crystallographic coordinates for the two phosducin (Pdc) template molecules were based on published crystal structures (PDB entry 1B9X, and 2TRC). The sequence for mouse PhLP was taken from the SwissProt database (accession number Q9DBX2). Sequence alignment and generation of a 3D model for PhLP was accomplished using INSIGHTII/CONSENSUS software (Accelrys, San Diego, CA, USA) v.2005 on a Silicon Graphics workstation. All simulations were conducted at the Computational Chemistry and Molecular Modeling Lab as part of the WVU Health Science Center.

Sequence alignment - Sequence alignment and the generation of a 3D model of PhLP1 was accomplished using the HOMOLOGY module of INSIGHTII (Accelrys, San Diego, CA, USA). The sequences for the two Pdc template molecules were first aligned by identifying structurally conserved regions (SCRs). SCRs are based on root-mean-square (rms) deviation of the backbone and were determined by maximizing the scores from Dayhoff's mutation matrix and the Engleman and Steitz hydrophobicity index. After SCRs for the template molecules were assigned, PhLP was then aligned to the template sequence using manual pairwise-alignment. Secondary structural elements were predicted based on primary amino acid sequences using the web-based online tool, PredictProtein http://www.predictprotein.org/. The locations of each predicted secondary structural element on PhLP was then compared to those on Phosducin based on the sequence alignment. 
Generating the PhLP structure - After the SCRs were determined, coordinates of the model were assigned based on distance geometry calculations through the CONSENSUS module of INSIGHTII. The PhLP structure that was generated was refined using the BIOPOLYMER module of INSIGHTII to achieve the lowest energy conformation. 


\section{REFERENCES}

1. Munoz IG, Yebenes H, Zhou M, Mesa P, Serna M, Park AY, Bragado-Nilsson E, Beloso A, de Carcer G, Malumbres M, Robinson CV, Valpuesta JM, Montoya G. Crystal structure of the open conformation of the mammalian chaperonin CCT in complex with tubulin. Nat Struct Mol Biol. 2011 Jan; 18(1): 14-19.

2. Yebenes H, Mesa P, Munoz IG, Montoya G, Valpuesta JM. Chaperonins: Two rings for ing. Trends Biochem Sci. 2011 Aug; 36(8): 424-432.

3. Thulasiraman V, Yang CF, Frydman J. In vivo newly translated polypeptides are sequestered in a protected folding environment. EMBO J. 1999 Jan 4; 18(1): 85-95. PMCID: PMC1171105.

4. Spiess C, Meyer AS, Reissmann S, Frydman J. Mechanism of the eukaryotic chaperonin: Protein folding in the chamber of secrets. Trends Cell Biol. 2004 Nov; 14(11): 598-604. PMCID: PMC2812437.

5. Valpuesta JM, Martin-Benito J, Gomez-Puertas P, Carrascosa JL, Willison KR. Structure and function of a protein folding machine: The eukaryotic cytosolic chaperonin CCT. FEBS Lett. 2002 Oct 2; 529(1): 11-16.

6. Simonds WF. G protein-regulated signaling dysfunction in human disease. J Investig Med. 2003 Jul; 51(4): 194-214.

7. Schoneberg T, Schulz A, Biebermann H, Hermsdorf T, Rompler H, Sangkuhl K. Mutant G-protein-coupled receptors as a cause of human diseases. Pharmacol Ther. 2004 Dec; 104(3): 173-206.

8. Ditzel L, Lowe J, Stock D, Stetter KO, Huber H, Huber R, Steinbacher S. Crystal structure of the thermosome, the archaeal chaperonin and homolog of CCT. Cell. 1998 Apr 3; 93(1): 125-138.

9. Knol JC, Engel R, Blaauw M, Visser AJ, van Haastert PJ. The phosducin-like protein PhLP1 is essential for G\{beta\}\{gamma\} dimer formation in dictyostelium discoideum. Mol Cell Biol. 2005 Sep; 25(18): 8393-8400. PMCID: PMC1234308.

10. Humrich J, Bermel C, Bunemann M, Harmark L, Frost R, Quitterer U, Lohse MJ. Phosducin-like protein regulates Gprotein betagamma folding by interaction with tailless complex polypeptide-1alpha: Dephosphorylation or splicing of PhLP turns the switch toward regulation of gbetagamma folding. J Biol Chem. 2005 May 20; 280(20): 20042-20050.

11. Marrari Y, Crouthamel M, Irannejad R, Wedegaertner PB. Assembly and trafficking of heterotrimeric G proteins. Biochemistry. 2007 Jul 3; 46(26): 7665-7677. PMCID: PMC2527407.

12. Willardson BM, Howlett AC. Function of phosducin-like proteins in G protein signaling and chaperone-assisted protein folding. Cell Signal. 2007 Dec; 19(12): 2417-2427. PMCID: PMC2095786.

13. Schroder S, Lohse MJ. Quantification of the tissue levels and function of the G-protein regulator phosducin-like protein (PhlP). Naunyn Schmiedebergs Arch Pharmacol. 2000 Nov; 362(4-5): 435-439.

14. Thibault C, Sganga MW, Miles MF. Interaction of phosducin-like protein with G protein betagamma subunits. J Biol Chem. 1997 May 9; 272(19): 12253-12256.

15. Blaauw M, Knol JC, Kortholt A, Roelofs J, Ruchira, Postma M, Visser AJ, van Haastert PJ. Phosducin-like proteins in dictyostelium discoideum: Implications for the phosducin family of proteins. EMBO J. 2003 Oct 1; 22(19): 5047-5057.

PMCID: PMC204491.

16. Flanary PL, DiBello PR, Estrada P, Dohlman HG. Functional analysis of Plp1 and Plp2, two homologues of phosducin in yeast. J Biol Chem. 2000 Jun 16; 275(24): 18462-18469.

17. Stirling PC, Cuellar J, Alfaro GA, El Khadali F, Beh CT, Valpuesta JM, Melki R, Leroux MR. PhLP3 modulates CCTmediated actin and tubulin folding via ternary complexes with substrates. J Biol Chem. 2006 Mar 17; 281(11): 7012-7021.

18. Martin-Benito J, Bertrand S, Hu T, Ludtke PJ, McLaughlin JN, Willardson BM, Carrascosa JL, Valpuesta JM. Structure of the complex between the cytosolic chaperonin CCT and phosducin-like protein. Proc Natl Acad Sci U S A. 2004 Dec 14; 101(50): 17410-17415. PMCID: PMC536017. 
19. Miles MF, Barhite S, Sganga M, Elliott M. Phosducin-like protein: An ethanol-responsive potential modulator of guanine nucleotide-binding protein function. Proc Natl Acad Sci U S A. 1993 Nov 15; 90(22): 10831-10835. PMCID:

PMC47872.

20. Humrich J, Bermel C, Grubel T, Quitterer U, Lohse MJ. Regulation of phosducin-like protein by casein kinase 2 and Nterminal splicing. J Biol Chem. 2003 Feb 14; 278(7): 4474-4481.

21. Savage JR, McLaughlin JN, Skiba NP, Hamm HE, Willardson BM. Functional roles of the two domains of phosducin and phosducin-like protein. J Biol Chem. 2000 Sep 29; 275(39): 30399-30407.

22. Lukov GL, Hu T, McLaughlin JN, Hamm HE, Willardson BM. Phosducin-like protein acts as a molecular chaperone for $G$ protein betagamma dimer assembly. EMBO J. 2005 Jun 1; 24(11): 1965-1975. PMCID: PMC1142607.

23. Lambright DG, Sondek J, Bohm A, Skiba NP, Hamm HE, Sigler PB. The 2.0 A crystal structure of a heterotrimeric G protein. Nature. 1996 Jan 25; 379(6563): 311-319.

24. Lin Y, Smrcka AV. Understanding molecular recognition by G protein betagamma subunits on the path to pharmacological targeting. Mol Pharmacol. 2011 Oct; 80(4): 551-557. PMCID: PMC3187535.

25. Lukov GL, Baker CM, Ludtke PJ, Hu T, Carter MD, Hackett RA, Thulin CD, Willardson BM. Mechanism of assembly of $\mathrm{G}$ protein betagamma subunits by protein kinase CK2-phosphorylated phosducin-like protein and the cytosolic chaperonin complex. J Biol Chem. 2006 Aug 4; 281(31): 22261-22274.

26. Gaudet R, Bohm A, Sigler PB. Crystal structure at 2.4 angstroms resolution of the complex of transducin betagamma and its regulator, phosducin. Cell. 1996 Nov 1; 87(3): 577-588.

27. Li C, Wong WH. Model-based analysis of oligonucleotide arrays: Expression index computation and outlier detection. Proc Natl Acad Sci U S A. 2001 Jan 2; 98(1): 31-36. PMCID: PMC14539.

28. Posokhova E, Song H, Belcastro M, Higgins L, Bigley LR, Michaud NA, Martemyanov KA, Sokolov M. Disruption of the chaperonin containing TCP-1 function affects protein networks essential for rod outer segment morphogenesis and survival. Mol Cell Proteomics. 2011 Jan; 10(1): M110.000570. PMCID: PMC3013443.

29. Lem J, Applebury ML, Falk JD, Flannery JG, Simon MI. Tissue-specific and developmental regulation of rod opsin chimeric genes in transgenic mice. Neuron. 1991 Feb; 6(2): 201-210.

30. Kapur K, Xing Y, Ouyang Z, Wong WH. Exon arrays provide accurate assessments of gene expression. Genome Biol. 2007; 8(5): R82. PMCID: PMC1929160. 